\title{
Multiscale finite element analysis of wave propagation in periodic solids
}

\author{
F. Casadei ${ }^{\mathrm{a}, \mathrm{b}}$, J.J. Rimolia ${ }^{\mathrm{a}, *}$, M. Ruzzene ${ }^{\mathrm{a}, \mathrm{c}}$ \\ ${ }^{a}$ School of Aerospace Engineering, Georgia Institute of Technology, Atlanta (GA), 30332 \\ ${ }^{b}$ School of Engineering and Applied Sciences, Harvard University, Cambridge (MA), 02138 \\ ${ }^{c}$ School of Mechanical Engineering, Georgia Institute of Technology, Atlanta (GA), 30332
}

\begin{abstract}
This paper reports on the application of the geometric multiscale finite element method for the analysis of wave propagation in heterogeneous periodic solids. The proposed scheme exploits multi-node elements to describe the microstructure through a local, auxiliary mesh that resolves the fine scale features, and that is used to numerically compute a set of interpolation functions employed for elements formulations at the global level. The method is applied for the analysis of the dispersion properties of, and transient wave propagation in domains featuring periodicity in two dimensions. Band diagram calculations, wave velocities and time domain computations are conducted on solids discretized using two-dimensional and three-dimensional multiscale finite element meshes. Results for assemblies with periodic inclusions, phononic stubbed plates and structural lattices illustrate the effectiveness of the method. Accurate predictions of dispersion relations, wave modes and time domain simulations are obtained with significant reductions in model size. The presented examples also illustrate some of the interesting wave characteristics of the considered class of periodic structures, which include wave directionality and frequency bandgaps.
\end{abstract}

\footnotetext{
*Corresponding author (rimoli@gatech.edu)
} 
Keywords: Geometric multiscale finite element method, wave propagation, periodic solids, dispersion relations. 


\section{Introduction}

Extensive research is devoted to the analysis and design of periodic structures and metamaterials for acoustic waves management [1]. Such assemblies exhibit interesting wave propagation properties such as bandgaps $[1,2,3]$, response directionality [4, 5, 6], left-handedness [7], and negative acoustic refraction [8]. All of these features can be employed for the design of acoustic devices operating over a broad range of frequencies and length scales. The application of such concepts can, for example, be used to perform a variety of acoustics-based signal processing functions at frequencies where electronics suffer from severe power limitations. In conjunction to telecommunication and signal processing, potential implications of the "acoustic wave guiding" technology include among others active sensing of structural integrity [9], and dissipation of high frequency modes of vibration [10]. Other novel structural con urations may be exploited for devices which exhibit acoustic super-lensing, super-focusing and/or cloaking characteristics [7, 8].

Unusual wave properties of the kind mentioned above are associated with material and structural heterogeneities, which mostly correspond to periodic modulations of stiffness and inertial properties. Such modulations may result from the periodic dispersion of inclusions of different materials within a matrix $[1,5]$, or from the microstructural configuration of a given assembly $[6,11]$. The spatial scales of property and geometry modulations dictate the range of frequencies at which wave guiding occurs, and therefore determines the scale and frequency range of application of a device. Very often the size of the microstructure is comparable to the wavelength of the propagating wavefield. Hence, the discretization and simulation of such problems may result in extremely high computational costs as the numerical grid must resolve both the cell and the microstructure length scales. The need for a simple and 
reliable numerical scheme is also motivated by the emerging field of band structure optimization $[12,13]$ where numerous repeated calculations are required to converge toward a desired design configuration.

Many techniques have been developed to predict the relevant properties of heterogeneous structures and materials, without the need to fully resolve the smallest spatial scales. For instance, homogenization based methods have the objective of describing the overall behavior of an heterogeneous system in terms of equivalent properties $[14,15,16,17]$. For example, composite materials are classically analyzed in terms of effective properties based on rules of mixtures [18]. Also, periodic structural lattices have been studied through homogenization strategies whereby the spatial periodicity is exploited to obtain dynamic equilibrium equations in the Fourier domain $[19,20,21,22,23]$. Homogenization methods are known to provide accurate results if the macroscopic fields are constant or slowly varying within a single representative volume element. Typically, such requirements hold far from the boundaries of the computational domain and when the size of the microstructure is significantly smaller than the wavelength of deformation.

In order to overcome the limitations of homogenization-based techniques, significant research effort is focused on the development of higher order expansion methods capable of describing the high frequency response and the dispersive behavior of periodic media. For example, Fish et al. [24, 25, 26, 27, 28, 29] proposed a multi-grid solver for wave problems in heterogeneous media characterized by microstructures whose size is comparable to that of the structural details or the wavelength of a traveling signal. Similarly, Murakami et al. [30, 31, 32] proposed a mixed theory based on high-order continuum models to simulate elastic wave dispersion in heterogeneous composite media. More recently, a reduced Bloch mode expansion method has been introduced by Hussein [33] for the calculation of band diagrams of periodic solids. 
The method, based on a FE discretization of the unit cell, employs a limited number of Bloch eigenmodes to project the fine-scale eigenvalue problem onto a reduced subspace selected within the irreducible Brillouin zone at high symmetry points. Being in line with the well known concept of modal analysis, the approach maintains accuracy while reducing the computation time even if the calculation of the reduced basis requires explicit solution of the local eigenvalue problem. The idea of using a set of complex Bloch eigenmodes to formulate a specialized multiscale FEM solver is also found in the work by Brandsmeier et al. [34].

Despite the outstanding achievements in the field of high-order homogenization, efficient computation of dispersion and the simulation of wave propagation in heterogeneous media is still a considerable challenge. In this paper, the geometric multiscale finite element method (GMsFEM) recently developed by the authors [35] is applied to the analysis of wave propagation in heterogeneous periodic media. In is worth mentioning that this approach was already shown to be precise and computationally efficient for the analysis of elastic wave scattering from localized defects [36]. The approach is based on the formulation of multiscale elements (MSEs) with an arbitrary number of nodes that are used to model heterogeneities occurring at sub-cell length scales. In contrast to other multiscale strategies $[33,34]$ that require the solution of a complex eigenvalue problem to construct the enrichment basis, the proposed multiscale shape functions are expressed in terms of a simple algebraic operator. In addition, the adopted numerical scheme can be also exploited for the explicit transient simulation of wave propagation in periodic domains of finite size.

The paper is organized into 6 sections including this introduction. Section 2 briefly reviews the background on wave propagation in periodic elastic solids, and Section 3 describes the multiscale finite element formulation providing details on the calculation of the multiscale shape functions and the MSE matrices. Sections 4 and 5 
illustrate the application of the GMsFEM for the calculation of dispersion relations, wave modes and time domain simulations involving two- and three-dimensional periodic solids. Finally, conclusions and future perspectives are summarized in section 6 .

\section{Elastic waves in periodic media}

The differential equations governing the motion of a heterogeneous medium can be expressed as:

$$
\nabla \cdot \sigma=\rho \ddot{\boldsymbol{u}}
$$

where $\boldsymbol{\sigma}$ is the stress tensor, $\boldsymbol{u}$ is the displacement vector, and $\rho$ denotes the material density. Under the assumption of linear elasticity, displacements and stresses are related by the material constitutive law and the strain-displacement relations:

$$
\boldsymbol{\sigma}=\boldsymbol{C}: \nabla^{s} \boldsymbol{u}
$$

where $\boldsymbol{C}$ is the symmetric material tensor, and $\nabla^{s}=\frac{1}{2}\left(\nabla \boldsymbol{u}+\nabla \boldsymbol{u}^{T}\right)$ is the symmetric part of the gradient operator. Substituting Eq. (2) into Eq. (1) gives:

$$
\nabla \cdot \boldsymbol{C}: \frac{1}{2}\left(\nabla \boldsymbol{u}+\nabla \boldsymbol{u}^{T}\right)=\rho \ddot{\boldsymbol{u}}
$$

which expresses the strong form of a general elastodynamic problem.

A periodic medium is characterized by the assembly of unit cells repeating in space according to a set of lattice vectors. For two dimensional (2D) periodic domains, for example, the position $\boldsymbol{r}_{p}$ of a generic point $P$ within cell $\left(n_{1}, n_{2}\right)$ can be expressed as:

$$
\boldsymbol{r}_{p}=\boldsymbol{r}_{p 0}+n_{1} \boldsymbol{e}_{1}+n_{2} \boldsymbol{e}_{2}
$$

where $\boldsymbol{r}_{p 0}$ is the position of point $P$ in the reference cell, $n_{1}, n_{2}$ are integers, and $\boldsymbol{e}_{1}, \boldsymbol{e}_{2}$ are the lattice vectors. 
For a periodic medium, the general solution of Eq. (3) can be expressed as a Bloch solution [37, 38, 33] given by:

$$
\boldsymbol{u}\left(\boldsymbol{r}_{p}, \boldsymbol{k}, t\right)=\widehat{\boldsymbol{u}}\left(\boldsymbol{r}_{p 0}, \boldsymbol{k}\right) e^{\left(\boldsymbol{k} \cdot \boldsymbol{r}_{p}-\omega t\right)}
$$

where $\widehat{u}\left(\boldsymbol{r}_{p 0}, \boldsymbol{k}\right)$ is the displacement Bloch function, which is periodic. Equation (5) expresses the solution of Eq. (3) in terms of a periodic function defined over a unit cell and of a phase operator, which describes the phase changes for a plane wave propagating from one cell to the next. Accordingly, wave motion in the periodic media is fully defined once the Bloch functions $\widehat{\boldsymbol{u}}\left(\boldsymbol{r}_{p 0}, \boldsymbol{k}\right)$ and the dispersion properties of the medium $\boldsymbol{k}=\boldsymbol{k}(\omega)$ are known. This information is obtained by substituting Eq. (5) into Eq. (3), which leads to the strong form of the Bloch eigenvalue problem:

$$
\nabla \cdot \boldsymbol{C}:\left[\nabla^{s} \widehat{\boldsymbol{u}}+\frac{1}{2} i\left(\boldsymbol{k}^{T} \otimes \widehat{\boldsymbol{u}}+\boldsymbol{k} \otimes \widehat{\boldsymbol{u}}^{T}\right)\right]=-\rho \omega^{2} \widehat{\boldsymbol{u}}
$$

Proper discretization of Eq. (6) through a weak formulation leads to a linear eigenvalue problem that can be solved in terms of Bloch modes and dispersion relations [33, 6]. Specifically, Eq. (6) is solved for the wave vector $\boldsymbol{k}$ spanning the range defining the first Brillouin zone. The calculated dispersion relations provide information on wave propagation characteristics, which include the presence of bandgaps, the anisotropy of the medium, and the wave velocities, and thus effectively characterize its dynamic behavior.

\section{Multiscale element formulation}

\subsection{Motivation and concept}

The discretized version of Eq. (6) can be obtained through a refined mesh of the unit cell, or using a reduced order modeling technique of the kind presented in this paper. Reductions in model size are driven by the need of decreasing the 
computational cost associated with the evaluation of the dispersion properties of the medium under consideration.

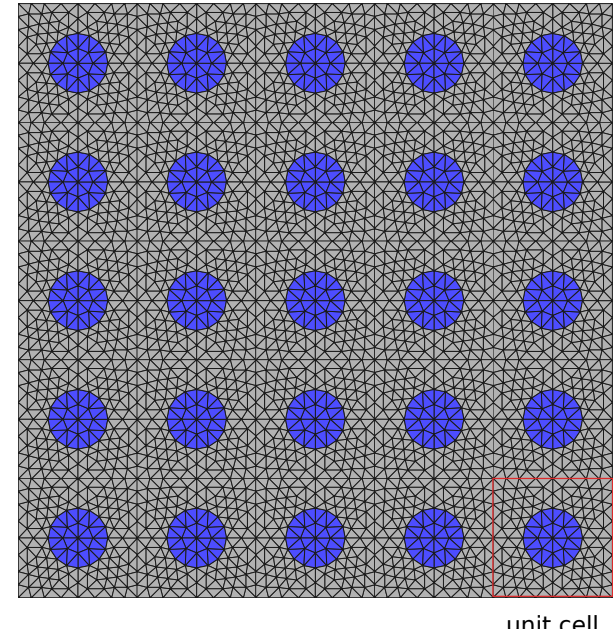

(a)

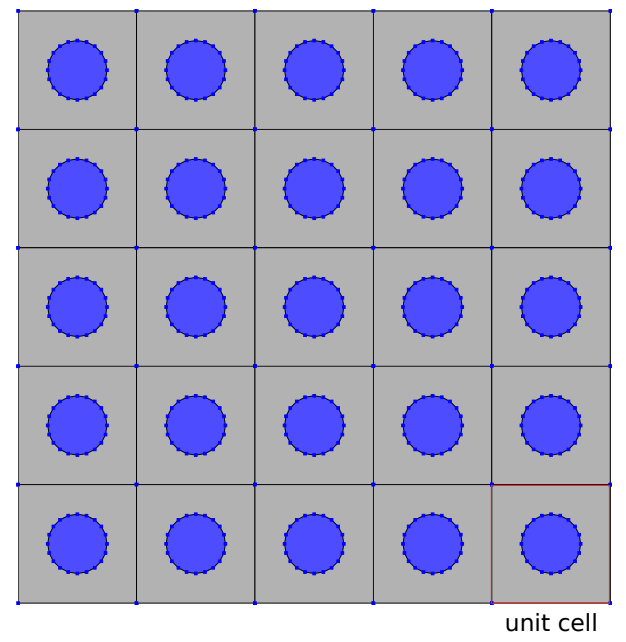

(b)

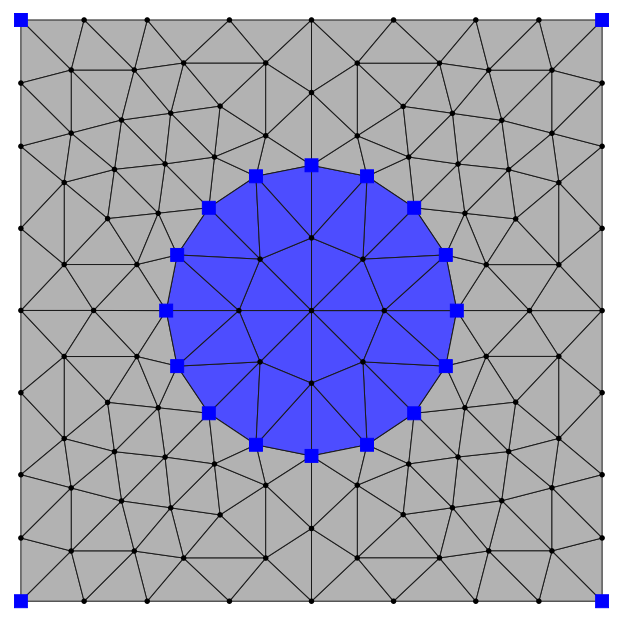

(c)

Figure 1: Periodic assembly with mesh driven by local heterogeneity (a), and MSE-based discretization (b). Detail of MSE of the unit cell with corresponding fine scale triangulation and partition between local $(\bullet)$ and global $(\boldsymbol{\square})$ nodes $(\mathrm{c})$. 
More importantly, reduced model sizes are highly desirable while performing dynamic simulations on finite domains consisting of the assembly of a large number of unit cells. In this context, the resolution of local features at the unit cell level imposes demanding requirements in terms of local mesh refinement, which leads to very large models when assemblies of several units are considered. This is illustrated schematically in Fig. 1, which shows a discretization at the unit cell level and its replication over a finite domain, potentially leading to a highly refined mesh and a correspondingly large model.

In the proposed numerical scheme, the behavior of the unit cell at the global level is described by a limited number of nodes, whose motion defines the degrees of freedom (DOFs) of the corresponding multiscale element (MSE). The MSE resolves geometrical features or material discontinuities within the unit cell without requiring localized mesh refinements. The nodal displacements of a MSE are interpolated within the element's domain by an auxiliary fine-scale mesh that discretizes the unit cell region. For example, Fig. 1.c shows the fine-scale triangulation of an MSE. The DOFs associated with such local mesh are denoted as fine scale DOFs $\boldsymbol{u}$. A subset of $\boldsymbol{u}$ is retained at the coarse-scale level and represents the coarse scale DOFs $\boldsymbol{d} \in \boldsymbol{u}$ of the multiscale element that are assembled at the structural level. Of note is that the fine scale mesh, and the associated local DOFs, is not retained as part of the macroscopic simulation, but it is only used to compute the MSE's multiscale shape functions as described in the following section.

\subsection{Numerical interpolation functions}

A set of multiscale interpolation functions is obtained through a numerical solution at the local level, and are subsequently used for the formulation of mass and stiffness matrices of the MSE. The interpolation functions $\mathcal{N}$ map the fine scale 
DOFs with the DOFs retained at the coarse scale through the following algebraic relation:

$$
\boldsymbol{u}=\mathcal{N} \boldsymbol{d}
$$

The vector of fine scale DOFs $\boldsymbol{u}$ is partitioned in terms of the coarse scale DOFs $\boldsymbol{d}$ previously defined, and a subset of local DOFs $\boldsymbol{q}$ that are not explicitly retained at the macroscopic level. This relation can be generally expressed as:

$$
\boldsymbol{u}=[\boldsymbol{d}, \boldsymbol{q}]^{T} \in \mathbb{R}^{n_{u} \times 1}
$$

where $\boldsymbol{d} \in \mathbb{R}^{n_{d} \times 1}$ and $\boldsymbol{q} \in \mathbb{R}^{n_{q} \times 1}$, with $n_{d}$ and $n_{q}$ respectively denoting the number of coarse scale DOFs and the number of discarded fine scale DOFs so that $n_{u}=n_{d}+n_{q}$. Substituting Eq. (8) into Eq. (7) leads to the following form for the interpolation matrix $\mathcal{N}$ :

$$
\mathcal{N}=\left[\begin{array}{c}
\boldsymbol{I}_{n_{d}} \\
\boldsymbol{\Gamma}
\end{array}\right]
$$

where $\boldsymbol{I}_{n_{d}} \in \mathbb{R}^{n_{d} \times n_{d}}$ is the identity matrix, while $\boldsymbol{\Gamma} \in \mathbb{R}^{n_{q} \times n_{d}}$ is a mapping operator that projects the coarse scale solution onto the DOFs $\boldsymbol{q}$ so that:

$$
q=\Gamma d
$$

The derivation of matrix $\Gamma$ relies on the numerical solution of an equilibrium problem performed at the local level, whereby the unit cell is discretized through a refined mesh, as illustrated in Fig. 1.c. Based on this discretization, the strain energy of the considered element can be expressed as:

$$
\mathcal{U}=\frac{1}{2} \boldsymbol{u}^{T} \boldsymbol{K}_{f} \boldsymbol{u}
$$

where $\boldsymbol{K}_{f}$ is the stiffness matrix corresponding to the local triangulation of Fig. 1.c. 
When assemblies of several unit cells are considered, the present formulation needs to ensure compatibility among neighboring MSEs. Specifically, compatibility must be enforced at the local scale of each multiscale element where the presence of "hanging nodes" at the boundaries (Fig. 2) can potentially lead to discontinuous displacements. In the present approach, this condition is imposed by requiring that the local DOFs undergo a linear variation along the MSE edges. This can be generally described by the following multipoint constraint:

$$
\mathcal{C}=\boldsymbol{B}_{d} \boldsymbol{d}+\boldsymbol{B}_{q} \boldsymbol{q}=\mathbf{0}
$$

where $\boldsymbol{B}_{d}$ and $\boldsymbol{B}_{q}$ are known matrices. Eq. (12) is enforced at the element level through the definition of the following augmented strain energy functional:

$$
\mathcal{U}^{+}=\mathcal{U}+\lambda^{T} \mathcal{C}
$$

where $\boldsymbol{\lambda}$ is a vector of Lagrange multipliers which enforce the variation of local DOFs to match the constraints imposed by the MSE shape functions [39].

unit cell 1

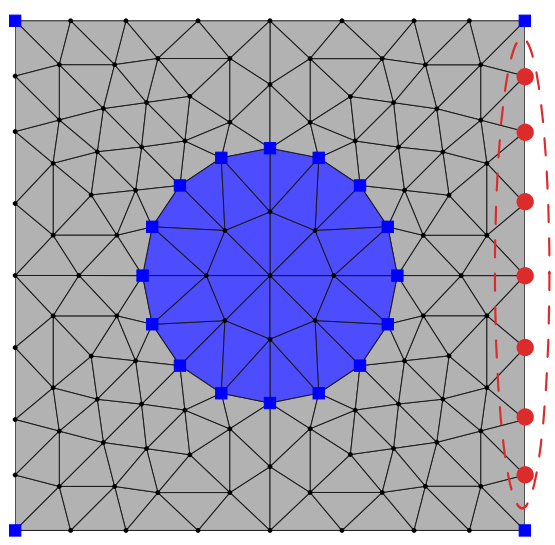

unit cell 2

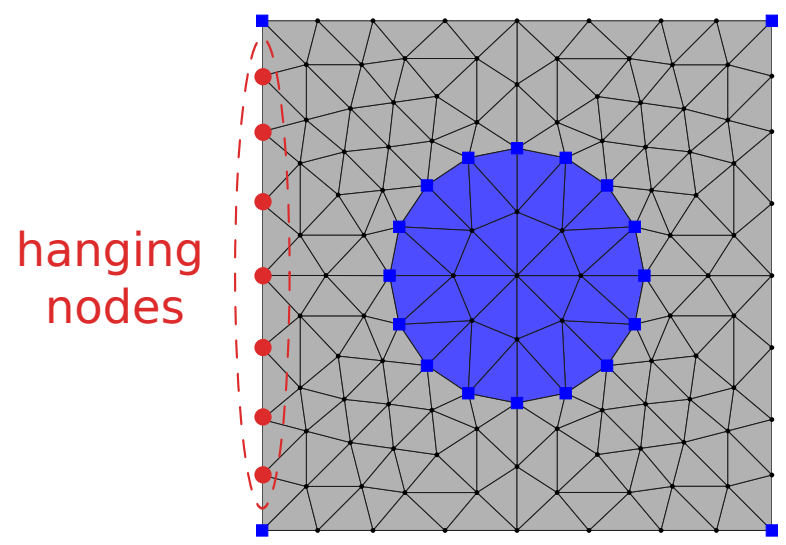

Figure 2: Hanging nodes (red dots) between adjacent multiscale elements. 
The stationary of $\mathcal{U}^{+}$with respect to the coarse scale DOFs $\boldsymbol{d}$, the fine scale DOFs $\boldsymbol{q}$, and the Lagrange multipliers $\boldsymbol{\lambda}$ is imposed by letting:

$$
\frac{\partial \mathcal{U}^{+}}{\partial \boldsymbol{d}}=\mathbf{0}, \quad \frac{\partial \mathcal{U}^{+}}{\partial \boldsymbol{q}}=\mathbf{0}, \quad \frac{\partial \mathcal{U}^{+}}{\partial \boldsymbol{\lambda}}=\mathbf{0}
$$

which leads to the following algebraic system of equations:

$$
\left[\begin{array}{ccc}
\boldsymbol{K}_{d d} & \boldsymbol{K}_{d q} & \boldsymbol{B}_{d}^{T} \\
\boldsymbol{K}_{d q}^{T} & \boldsymbol{K}_{q q} & \boldsymbol{B}_{q}^{T} \\
\boldsymbol{B}_{d} & \boldsymbol{B}_{q} & \mathbf{0}
\end{array}\right]\left[\begin{array}{c}
\boldsymbol{d} \\
\boldsymbol{q} \\
\boldsymbol{\lambda}
\end{array}\right]=\left[\begin{array}{l}
\mathbf{0} \\
\mathbf{0} \\
\mathbf{0}
\end{array}\right]
$$

Equation (15) enables the derivation of a relation between coarse and fine scale DOFs. For instance, from Eq. (15), it is possible to express $\boldsymbol{q}$ in terms of $\boldsymbol{d}$, by first substituting $\boldsymbol{\lambda}$. This gives:

$$
\boldsymbol{q}=-\boldsymbol{K}_{q q}^{-1}\left[\boldsymbol{K}_{d q}^{T}+\boldsymbol{B}_{q}^{T}\left(\boldsymbol{B}_{q} \boldsymbol{K}_{q q}^{-1} \boldsymbol{B}_{q}^{T}\right)^{-1}\left(\boldsymbol{B}_{d}-\boldsymbol{B}_{q} \boldsymbol{K}_{q q}^{-1} \boldsymbol{K}_{d q}^{T}\right)\right] \boldsymbol{d}=\boldsymbol{\Gamma} \boldsymbol{d}
$$

Equation (16) defines the interpolation operator $\boldsymbol{\Gamma}$ introduced in Eq. (10) as the linear map between the coarse scale DOFs $\boldsymbol{d}$ and the auxiliary local DOFs $\boldsymbol{q}$. Substituting Eq. (16) into Eq. (9) allows expressing the interpolation matrix $\mathcal{N}$ as:

$$
\mathcal{N}=\left[\begin{array}{c}
\boldsymbol{I}_{n_{d}} \\
-\boldsymbol{K}_{q q}^{-1}\left[\boldsymbol{K}_{d q}^{T}+\boldsymbol{B}_{q}^{T}\left(\boldsymbol{B}_{q} \boldsymbol{K}_{q q}^{-1} \boldsymbol{B}_{q}^{T}\right)^{-1}\left(\boldsymbol{B}_{d}-\boldsymbol{B}_{q} \boldsymbol{K}_{q q}^{-1} \boldsymbol{K}_{d q}^{T}\right)\right]
\end{array}\right]
$$

Equations 16 and 17 are valid as long as the matrix $\boldsymbol{K}_{q q}$ is not singular. For $n$ dimensional problems, this is always true as long as there are at least $n$ coarse-scale nodes within the MSE. Of note is the fact that this interpolation is obtained in terms of the local mechanical properties of the unit cell, with interelement compatibility explicitly enforced. 


\subsection{Multiscale element matrices}

The set of interpolation functions expressed by Eq. (17) can be employed for the formulation of the considered MSE to be assembled as part of a finite structural system. It is worth mentioning that when all unit cells are identical, as in the case of periodic systems of the kind considered herein, the formulation of the MSE needs to be conducted only once, so that the MSE of interest is available to be assembled as part of generic periodic arrays and for different types of numerical solutions.

For example, discretized forms of the equations of motion of periodic heterogeneous solids may be formulated by employing the Lagrangian of the system:

$$
\mathcal{L}=\mathcal{K}+\mathcal{U}-\mathcal{W}
$$

where $\mathcal{K}, \mathcal{U}, \mathcal{W}$ are the kinetic energy, the strain energy and work of the external forces acting on the considered unit cell. Based on the derivations above, the unit cell can be discretized by means of only one MSE so that the Lagrangian of the unit cell can be expressed in terms of the fine scale auxiliary mesh as:

$$
\mathcal{L}=\frac{1}{2} \boldsymbol{u}^{T} \boldsymbol{K}_{f} \boldsymbol{u}+\frac{1}{2} \dot{\boldsymbol{u}}^{T} \boldsymbol{M}_{f} \dot{\boldsymbol{u}}-\boldsymbol{f}_{f}^{T} \boldsymbol{u}
$$

Substituting the local-to-global interpolation defined in Eq. (7) gives:

$$
\begin{aligned}
\mathcal{K} & =\frac{1}{2} \dot{\boldsymbol{d}}^{T} \mathcal{N}^{T} \boldsymbol{M}_{f} \mathcal{N} \dot{\boldsymbol{d}}=\frac{1}{2} \dot{\boldsymbol{d}}^{T} \boldsymbol{M} \dot{\boldsymbol{d}} \\
\mathcal{U} & =\frac{1}{2} \boldsymbol{d}^{T} \mathcal{N}^{T} \boldsymbol{K}_{f} \mathcal{N} \boldsymbol{d}=\frac{1}{2} \boldsymbol{d}^{T} \boldsymbol{K} \boldsymbol{d} \\
\mathcal{W} & =\boldsymbol{f}_{f}^{T} \mathcal{N} \boldsymbol{d}=\boldsymbol{f}^{T} \boldsymbol{d}
\end{aligned}
$$

where $\boldsymbol{K}=\mathcal{N}^{T} \boldsymbol{K}_{f} \mathcal{N}$ and $\boldsymbol{M}=\mathcal{N}^{T} \boldsymbol{M}_{f} \mathcal{N}$ respectively denote the MSE stiffness and mass matrices, and $\boldsymbol{f}=\boldsymbol{\mathcal { N }}^{T} \boldsymbol{f}_{f}$ is a generalized load vector. The resulting matrices $\boldsymbol{K}$ and $\boldsymbol{M}$ represent a global system of equations to be employed for various types of dynamic analyses. Examples are provided in the following sections. 


\section{Band structure calculations for dispersion analysis}

The first application of the method considers the evaluation of the dispersion properties of periodic media. Band diagrams and wave modes are obtained through the application of Bloch theorem, which in its discretized form of Eq. (6) leads to the following eigenvalue problem:

$$
\left[\boldsymbol{K}(\boldsymbol{k})-\omega^{2} \boldsymbol{M}(\boldsymbol{k})\right] \boldsymbol{d}=\mathbf{0}
$$

where $\boldsymbol{k}$ is the wave vector. In Eq. (21), matrices $\boldsymbol{K}$ and $\boldsymbol{M}$ refer to a single unit cell, and typically correspond to a single MSE. In this case, the application of the multiscale formalism described in this study leads to some reduction in computational costs, but it is not as compelling as in the case of time domain simulations of assemblies of multiple unit cells. In fact, the computational cost associated with band structure computations is not particularly high. However, these calculations provide good benchmarks for the evaluation of the performance of the method, whereby the estimations obtained using the fine scale auxiliary mesh are compared with the predictions of MSEs with various number of nodes. This allows the direct comparison of the performance of the MSEs against a fine mesh solution used as a reference.

The two configurations considered in this section are two examples of phononic crystals, characterized by bandgaps generated by the periodic modulation of their acoustic impedance. In the first example, these periodic modulations are introduced by an array of inclusions within a matrix, while in the second case, periodic stubs mounted on an elastic plate lead to the generation of bandgaps within the Lamb wave spectrum $[40,41,42,43,44]$. In both cases, the periodic modulations produce scattering of waves at wavelengths that are of the order of the characteristic size of

the unit cell. This leads to the formation of bandgaps through Bragg scattering [45]. 


\subsection{Two-dimensional phononic crystal}

The configuration considered in this example consists of a 2D lattice of circular inclusions dispersed in a matrix of isotropic material $\left(E=71 \mathrm{GPa}, \rho=2700 \mathrm{~kg} / \mathrm{m}^{3}\right.$, and $\nu=0.3)$. The material of the inclusions is purely theoretical and has Young's modulus and density which are both ten times higher than in the matrix. The unit cell, shown in Fig. 3.a, is a square of side $L=1.0 \mathrm{~m}$, and the central circular inclusion has radius $R=0.25 L$.

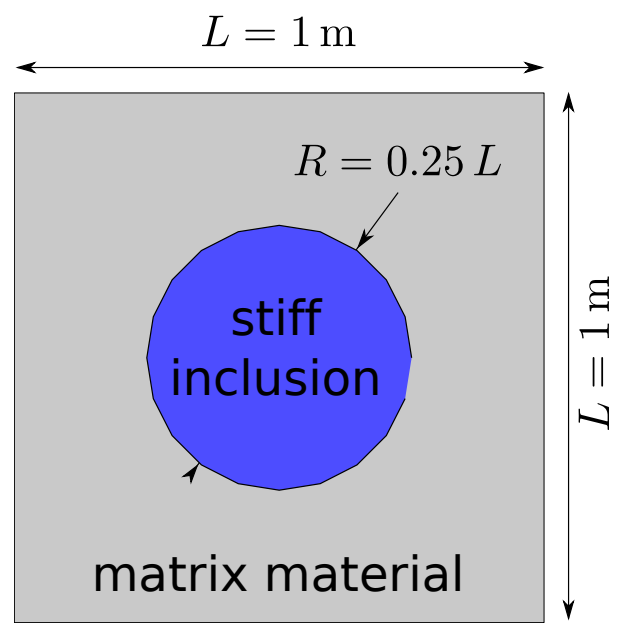

Figure 3: Unit cell geometry.

A fine mesh discretization is first considered as a reference. The mesh of Fig. 4.a consists of 1857 nodes belonging to triangular plane elements. The mesh is refined enough to describe the inclusions circular boundary, and it provides converged estimations of the first five dispersion branches. Given the dimensions and considered material properties, the frequency upper limit for this convergence analysis is $3.5 \mathrm{kHz}$. The dispersion properties of the system are also computed using the GMsFEM which allows discretizing the unit cell with a single MSE. In order to estimate the accuracy and convergence properties of the method, analyses are conducted using four differ- 
ent multiscale elements (denoted as MSE-A to MSE-D), with an increasing number of global nodes (Fig. 4.(b)-(e)) while keeping the same fine scale mesh.

Results of band structure calculations are shown in Fig. 5. As previously indicated, the frequency range of analysis is arbitrarily set to include the first 5 wave modes. Figure 5.a shows excellent agreement between the refined discretization (solid line) and the MSE formulation of Fig. 4.b for the first two dispersion branches. The first two wave modes are in fact characterized by deformation patterns that are well captured by the coarse MSE discretization. The results also highlight discrepancies on the high frequency modes, typically characterized by higher order deformations within the unit cell. In this case the compatibility conditions imposed along the MSE's boundaries tend to over-stiffen the model thus reducing its accuracy on wave modes and associated frequencies. Figures 5.c-e illustrate that an improved representation of the higher frequency branches is achieved by increasing the number of global nodes comprising the MSE. This allows capturing the deformation patterns typical of higher frequency modes. This interpretation is further supported by results shown in Fig. 6 that illustrate the eigenmodes computed for wave vector corresponding to point $\mathrm{M}$ of the irreducible Brillouin Zone, i.e. $\boldsymbol{k}=[\pi / L, \pi / L]$. The results show that the first eigenmode computed with the coarse MSE of Fig. 4.a are in good agreement with the corresponding refined mesh solutions, while discrepancies are observed for modes characterized by complex deformation patterns within the cell. Such discrepancies are reduced when employing the richer MSEs discretization of Fig. 4.d, which is capable to better resolve localized deformation patterns within the unit cell.

This example shows how the GMsFEM allows for a significant reduction of the model size by introducing multiscale elements featuring a limited number of global nodes. Despite band structure calculations are performed over a single cell, reduction 


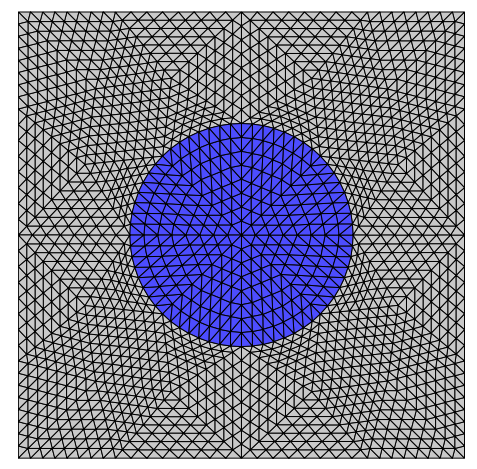

(a) FEM discretization

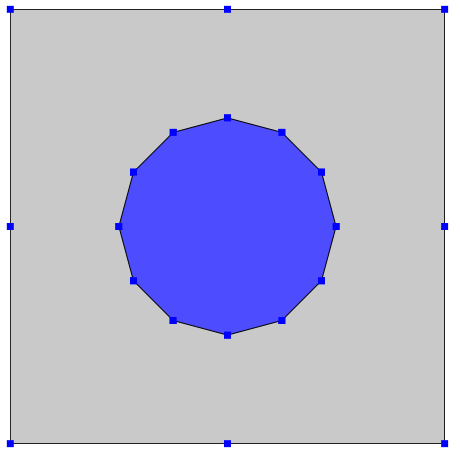

(b) MSE-A: 20 nodes

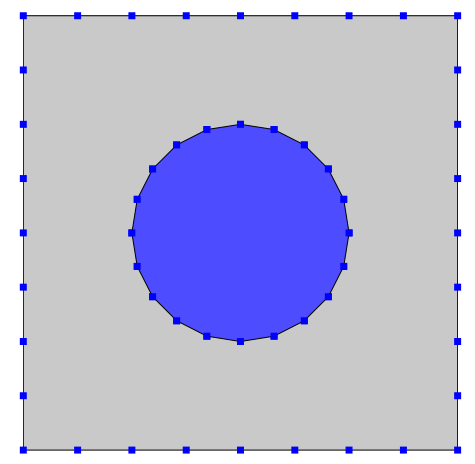

(d) MSE-C: 52 nodes

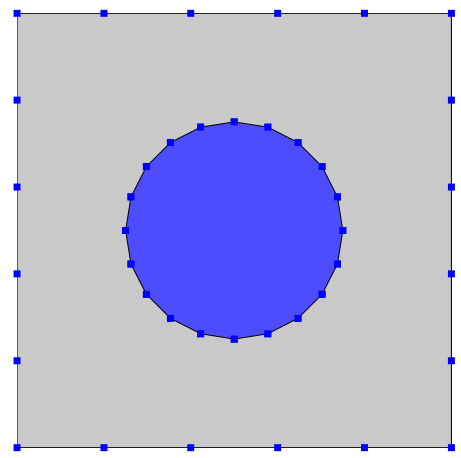

(c) MSE-B: 40 nodes

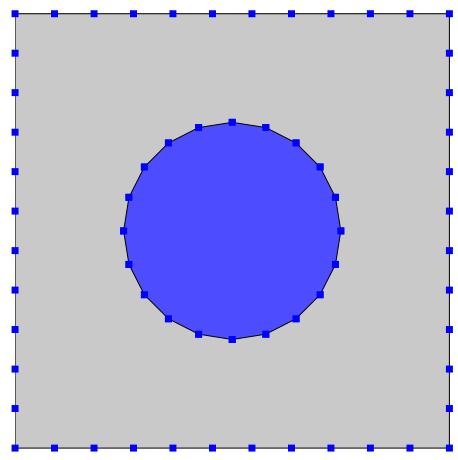

(e) MSE-D: 64 nodes

Figure 4: FE mesh employed as a reference for the band structure calculation (a), and multiscale elements used to discretize the considered unit cell (b)-(e). 


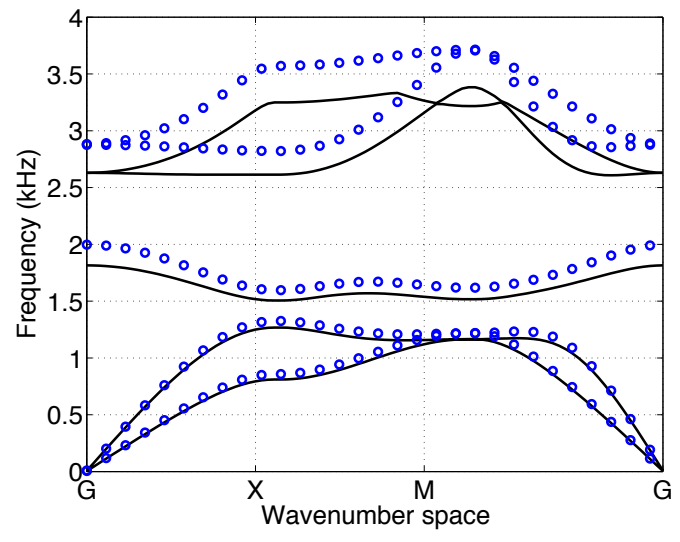

(a) MSE-A

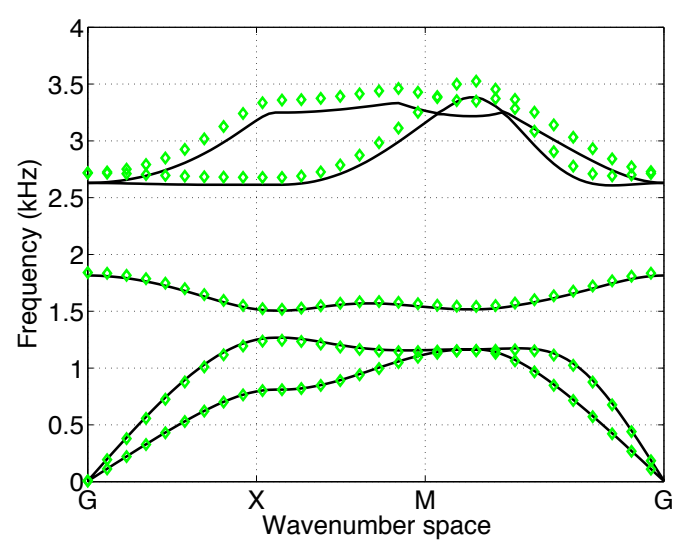

(c) MSE-C

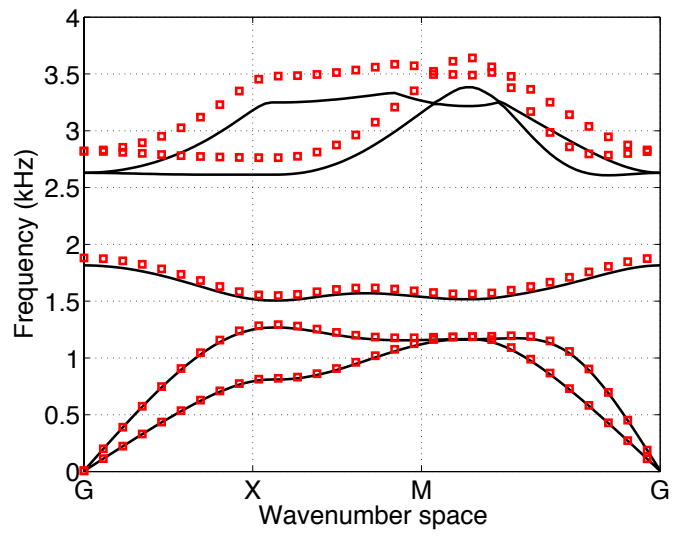

(b) MSE-B

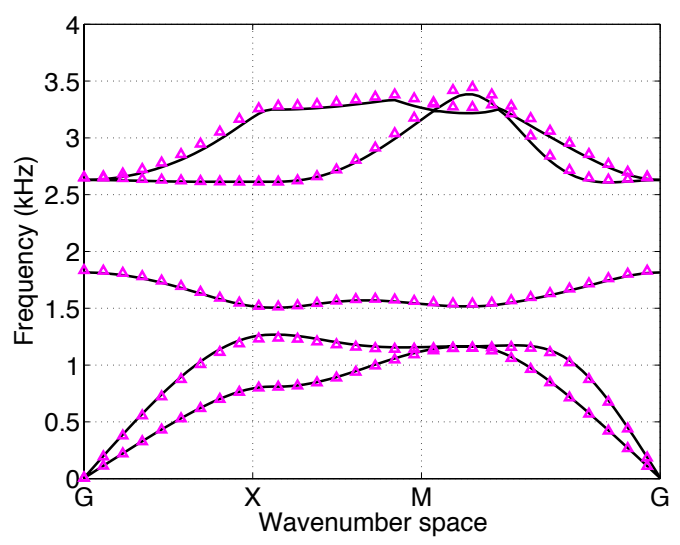

(d) MSE-D

Figure 5: Dispersion relations computed with GMsFEM (markers) using MSE-A (a), MSE-B (b), MSE-C (c), and MSE-D (d), and comparison with the reference FEM solution (thick solid line).

of the model size is critical since Bloch's eigenvalues need to be evaluated multiple times for different wavevectors spanning the contour of the Brillouin zone [33].

Interestingly, multiscale elements also allow for multiple cells to be combined into a larger model of finite size typically used for transient or steady state simulations of periodic structures. In this scenario, clearly, the need for a reduced model is 


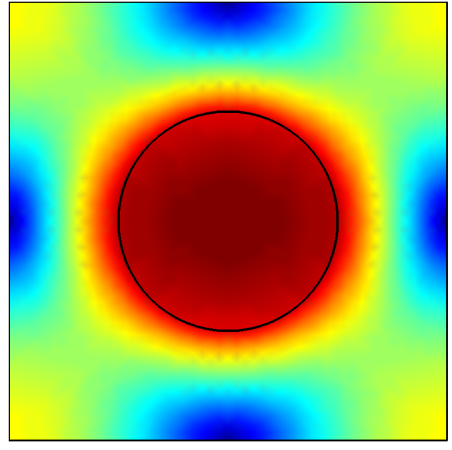

(a) Mode 1 (FEM)

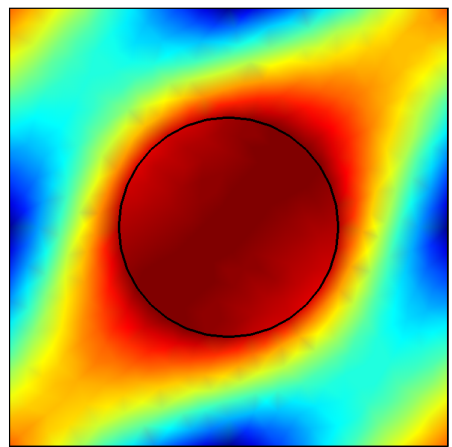

(d) Mode 1 (MSE-A)

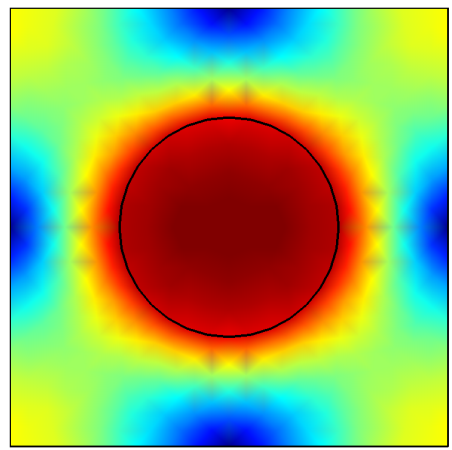

(g) Mode 1 (MSE-D)

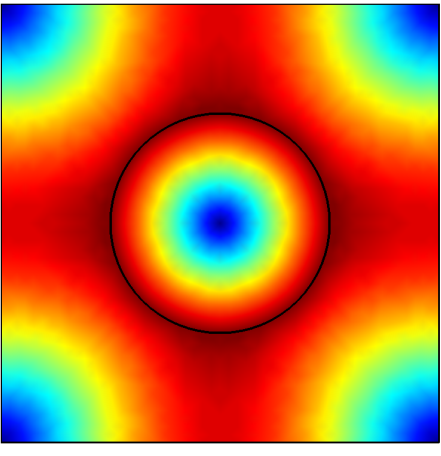

(b) Mode 3 (FEM)

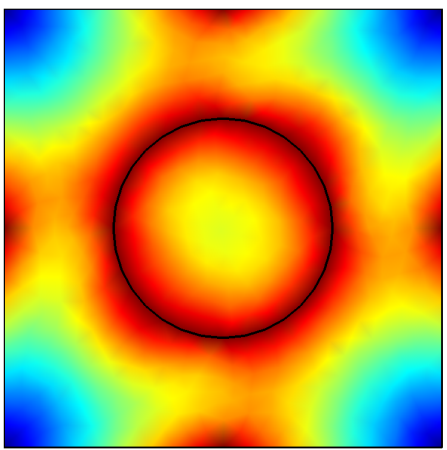

(e) Mode 3 (MSE-A)

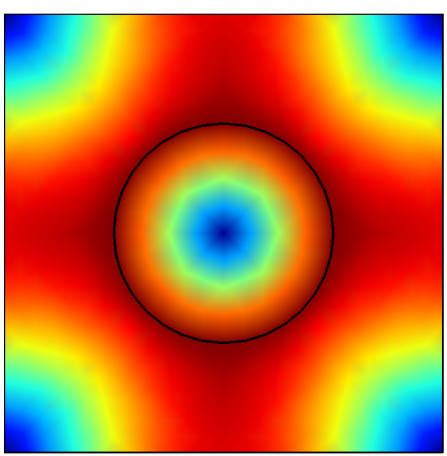

(h) Mode 3 (MSE-D)

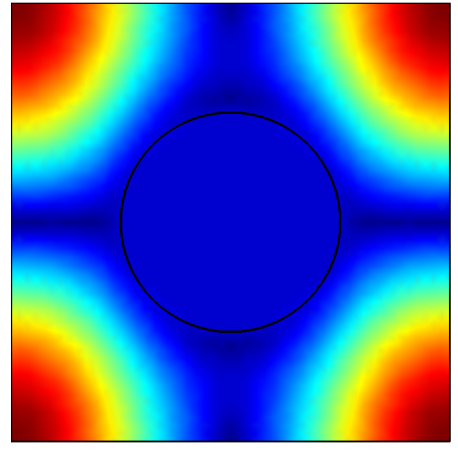

(c) Mode 5 (FEM)

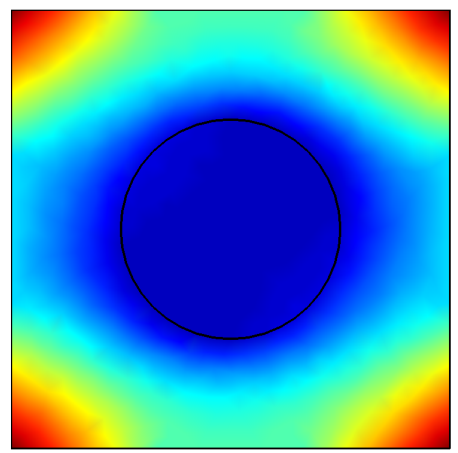

(f) Mode 5 (MSE-A)

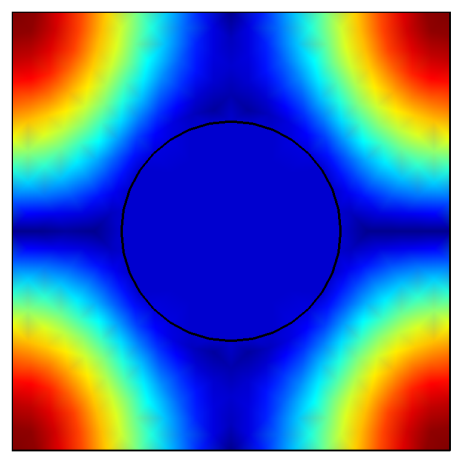

(i) Mode 5 (MSE-D)

Figure 6: Bloch eigenvectors (normalized amplitude) computed for $\boldsymbol{k}=\frac{\pi}{L}\left[\begin{array}{ll}1 & 1\end{array}\right]$ using the refined FEM mesh (a,b,c), MSE-A (d,e,f), and MSE-D (g,h,i). 
even more important since the size of the computational problem increases with the number of cells included in the domain. Here we illustrate this concept by analyzing wave propagation in a one-dimensional waveguide (see Fig. 7a) realized by removing one row of inclusions from a $7 \times 5$ array of the unit cells shown in Fig. 3. The system is excited by a point harmonic force located on the left side of the waveguide (Fig. 7a) at a frequency $\Omega=2 \mathrm{kHz}$ and the steady state amplitude of the response is computed both with the FEM and GMsFEM. Since in this frequency range wave propagation is forbidden in the periodic medium due to the presence of a complete bandgap (see Fig. 5), elastic waves are expected to propagate only inside the waveguide. Here, the GMsFEM discretization of the domain is realized by means of 35 MSEs, each modeling a singe unit cell, resulting in a total of 574 global DOFs. For comparison, an analogous FEM solution is computed by explicitly resolving the fine scale mesh used within each MSE (shown in Fig. 7a) over the entire domain. Therefore, the need for explicitly resolving both the global scale and the local scale of periodicity results in a much more refined model comprising 4017 global DOFs. The result of this analysis is shown in Fig. 7a,b showing the excellent agreement between the steady state amplitude of the displacement field computed with the two numerical schemes. Remarkably, the GMsFEM provides the same solution of the direct FE simulation at a fraction of the computational cost. Furthermore, the advantage of the proposed multiscale scheme is even more significant when a larger model is considered. This is shown in Fig. 8 which quantifies the increase of the relative model size, defined as the ratio between the number of DOFs in the FE model $\left(N_{F E M}\right)$, and the GMsFEM model $\left(N_{G M S}\right)$, as a function of the number of cells comprising the finite size domain. 


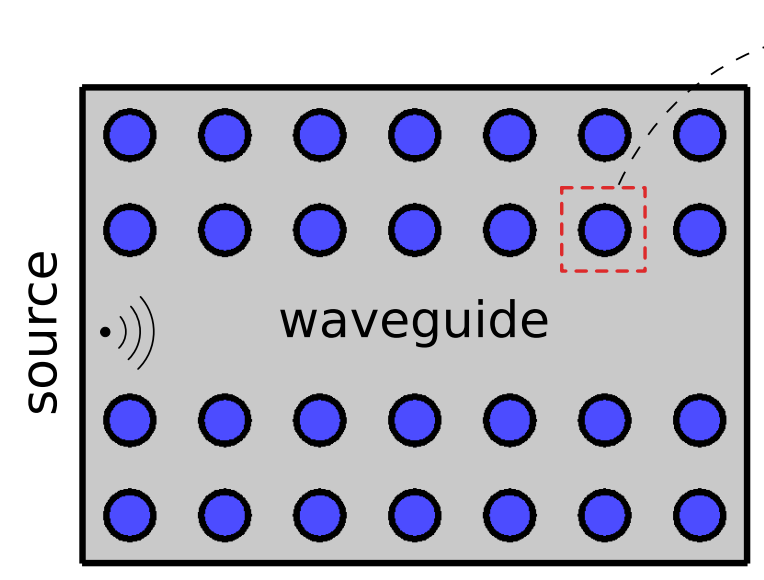

unit cell mesh

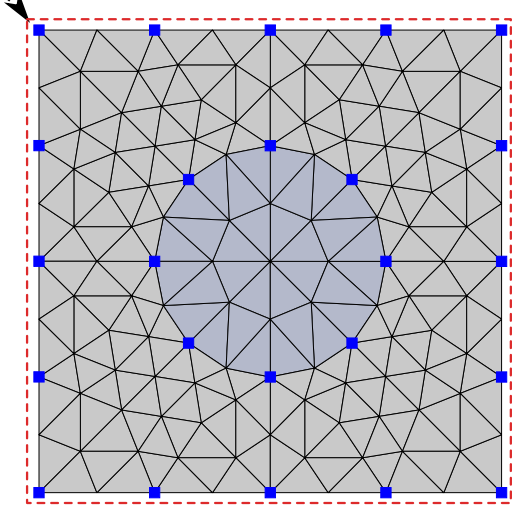

(a) Schematic of the configuration

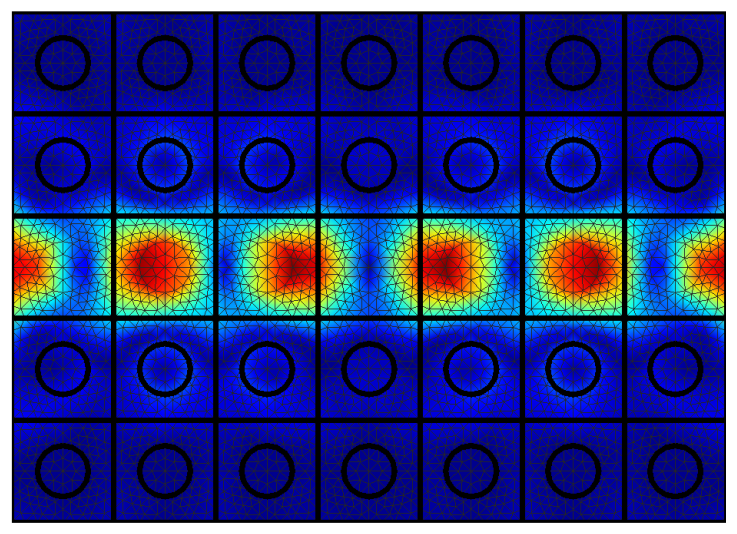

(b) GMsFEM solution

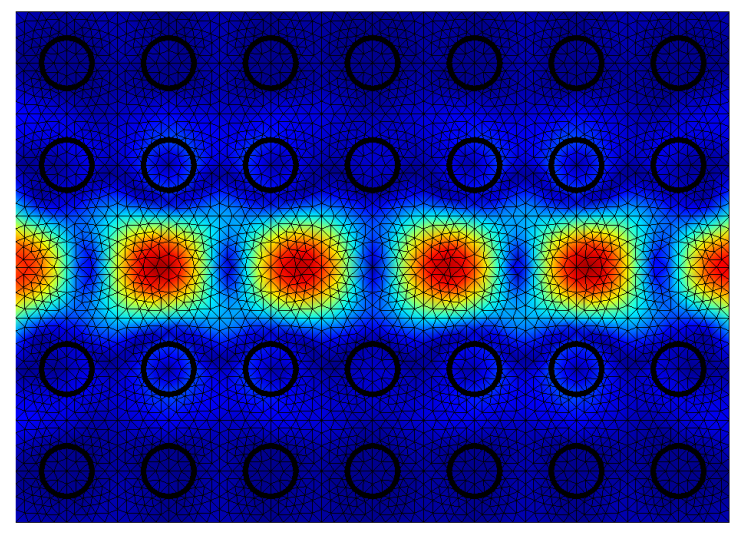

(c) FEM solution

Figure 7: Schematic of the finite size waveguide realized within the phononic crystal structure (a), and comparison between the FEM (b) and GMsFEM solutions (c).

\subsection{Periodic stubbed plates}

In this example the proposed method is applied for the calculation of the dispersion relations of a periodic stubbed plate. The considered structure, schematically shown in Fig. 9.a, consists of a $1 \mathrm{~mm}$ thick aluminum plate characterized by a periodic distribution of cylindrical stubs bonded to one surface. An interesting feature of this configuration, already investigated by $\mathrm{Wu}$ et al. [43], is the presence of a 


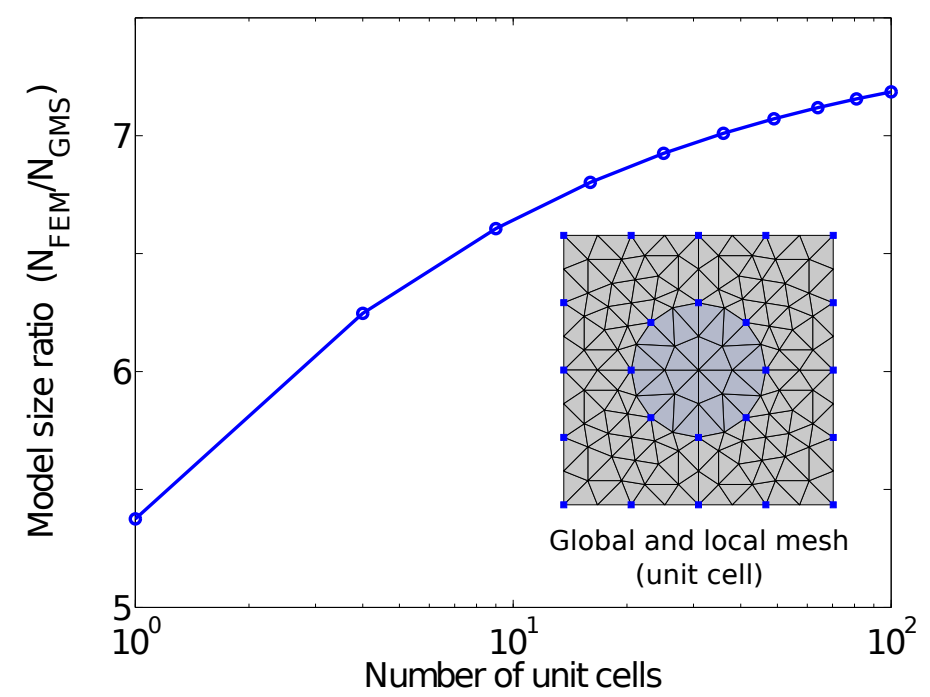

Figure 8: Relative model size as a function of the number of unit cells. The insert illustrates the local $(\bullet)$ and global $(\boldsymbol{\square})$ nodes over one unit cell.

complete bandgap that extends from 114 to $143 \mathrm{kHz}$, and two additional partial bandgaps centered at $21 \mathrm{kHz}$ and $110 \mathrm{kHz}$ respectively.

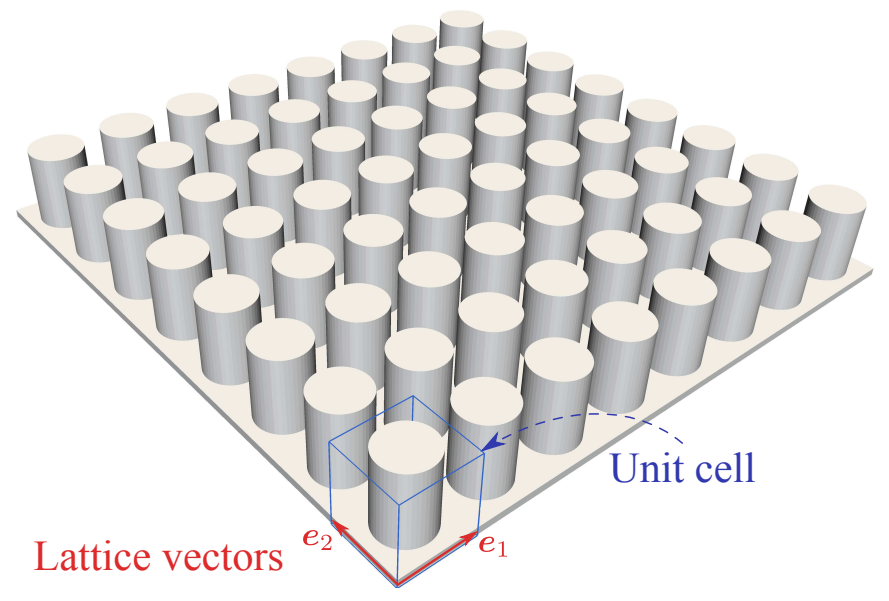

(a) Periodic stubbed plate

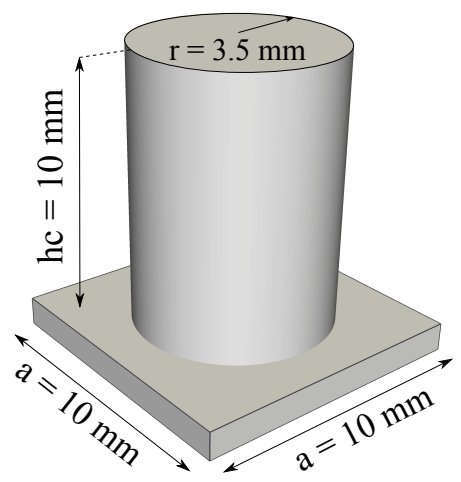

(b) Unit cell

Figure 9: Plate with periodic stubbed surface (a), and corresponding unit cell (b). 
As shown in Fig. 9.b the cylindrical stubs feature a diameter $d=7 \mathrm{~mm}$, height $h_{c}=10 \mathrm{~mm}$, and are arranged in a square lattice with a lattice constant $a=10 \mathrm{~mm}$. Although periodicity occurs only in a two-dimensional (2D) space defined by the lattices vectors $\left(\boldsymbol{e}_{1}, \boldsymbol{e}_{2}\right)$ in Fig. 9.a, the unit cell geometry requires the use of solid (3D) elements, which lead to a more challenging modeling scenario.

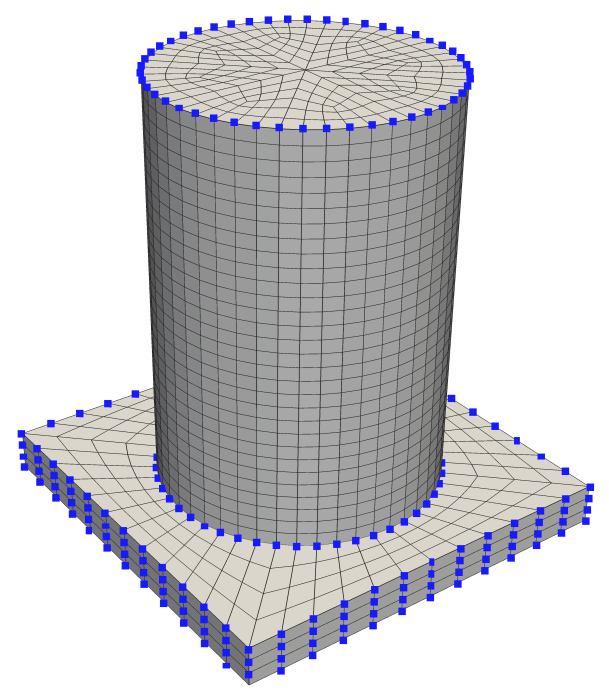

(a) MSE-A (288 global nodes)

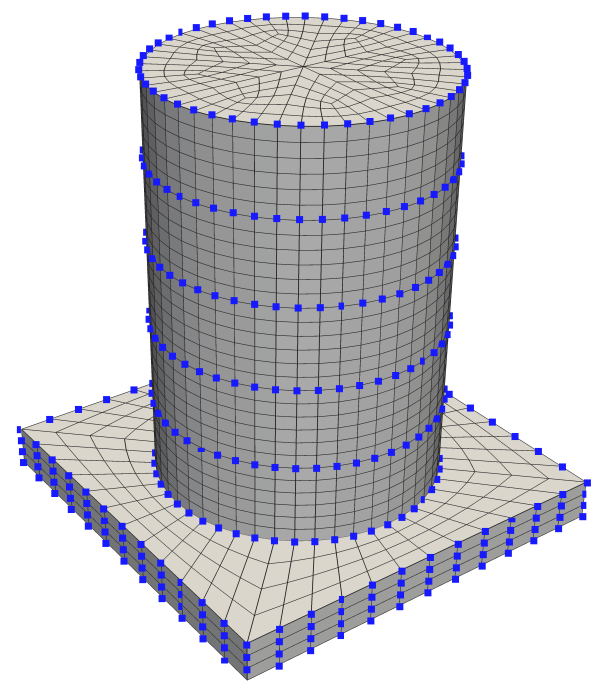

(b) MSE-B (480 global nodes)

Figure 10: Multiscale elements used to model the considered unit cell.

Two multiscale elements, shown in Fig. 10, are separately used to compute the dispersion relations of the unit cell. Each MSE is characterized by a different number of coarse scale nodes in order to assess the influence on the results. The dispersion relations are compared with a reference FE solution obtained by explicitly resolving the entire fine scale discretization comprising of 8962 nodes belonging to hexahedral elements (Fig. 10). The accuracy of such solution has been ascertained through a refinement study, and by comparison with the results presented in [43]. The band diagrams obtained with the coarser multiscale element (MSE-A) are shown in Fig. 11.a 
which illustrates how the MSE-A accurately predicts the dispersion relations of the three lower-frequency branches. Such wave modes include the fundamental symmetric $\left(S_{0}\right)$, anti-symmetric $\left(A_{0}\right)$, and shear horizontal $\left(S H_{0}\right)$ Lamb modes that propagate in the stubbed plate [43] that govern wave motion within the plate in a range up to $25 \mathrm{kHz}$.

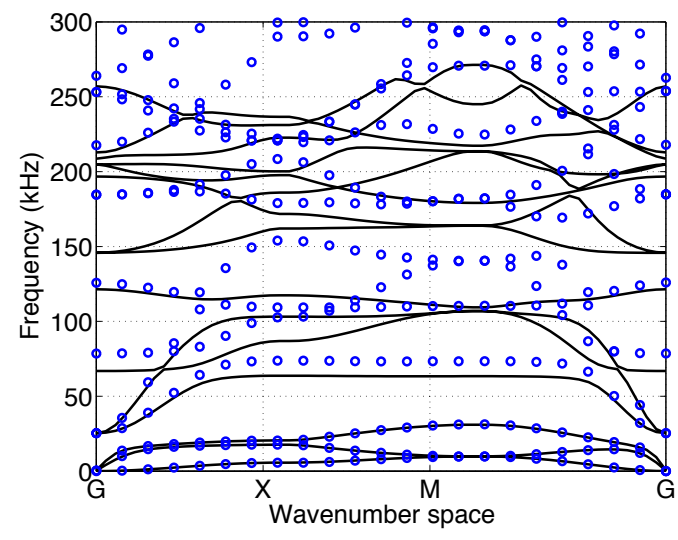

(a)

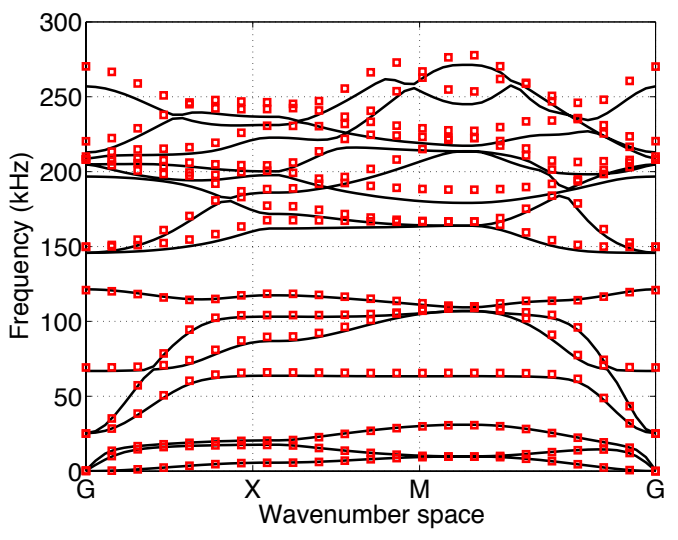

(b)

Figure 11: Comparison between the band diagrams computed with the FEM mesh (thick solid line) and the ones obtained using MSE-A (O) (a) and MSE-B ( $\square$ ) (b).

As shown in Fig. 12, these modes are characterized by a deformation pattern that can be properly captured by the MSE-A. Figure 11.a also shows that MSE-A does not accurately predict the high-frequency spectrum of the dispersion relations, which is instead better approximated by the refined MSE-B (Fig. 11.b). For instance, MSE-B provides an accurate description of the main bandgap of the system, even if minor discrepancies are observed above $200 \mathrm{kHz}$. A larger number of coarse scale nodes (Fig. 10.b) provides a better description of the dynamic deformation characteristics of these higher-frequency modes.

The examples presented in this section illustrate the proposed method and its 


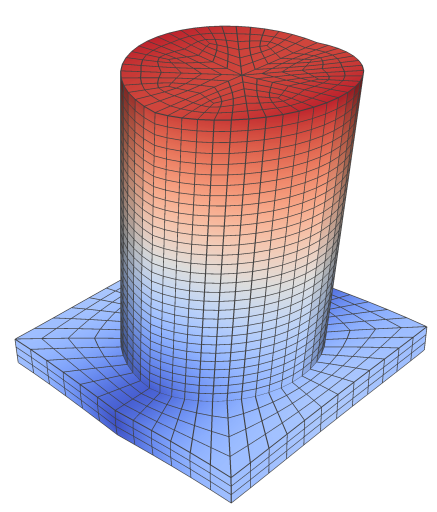

(a) Mode 1 (FEM)

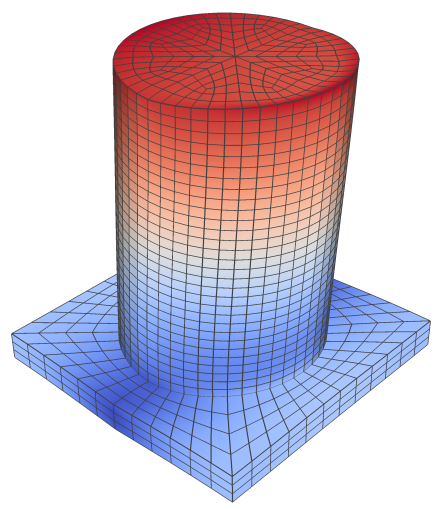

(d) Mode 1 (GMsFEM)

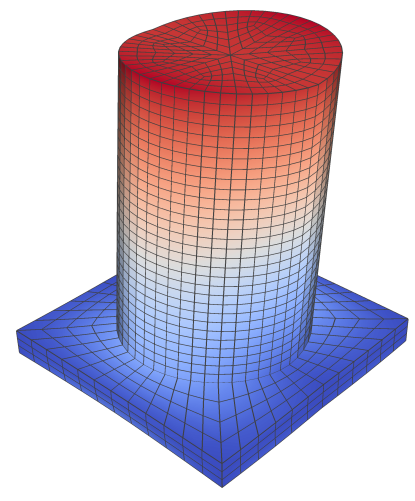

(b) Mode 2 (FEM)

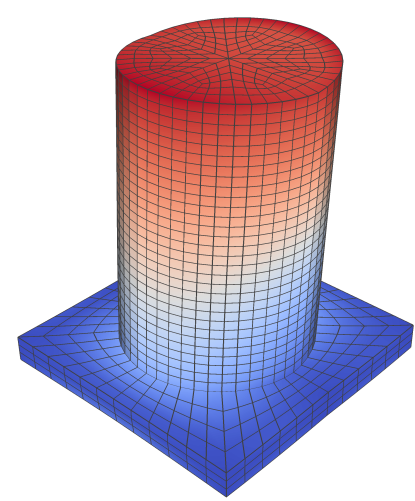

(e) Mode 2 (GMsFEM)

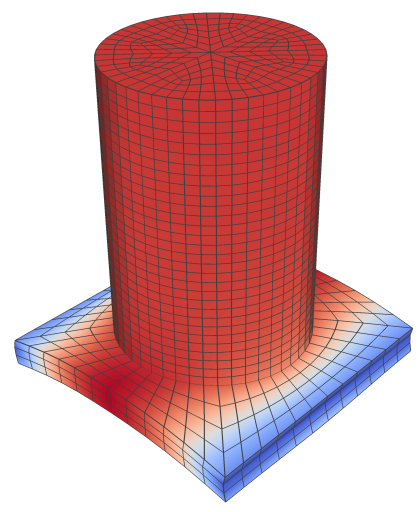

(c) Mode 3 (FEM)

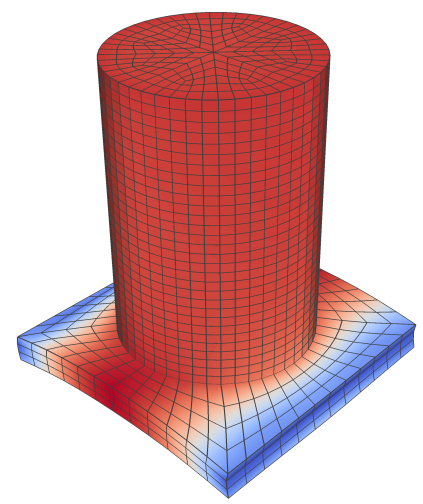

(f) Mode 3 (GMsFEM)

Figure 12: Bloch eigenmodes of the periodic plate computed for wave vector $\boldsymbol{k}=\frac{\pi}{a} \boldsymbol{i}_{1}$ using the FEM (a,b,c), and MSE-A (d,e,f).

ability to compute the dispersion relations of a periodic heterogeneous media using a one-element discretization of the unit cell. As expected, the results indicate that the number of global nodes defining the MSE dictates the corresponding frequency range of accuracy. This example also highlights that global nodes must be placed in relevant locations to capture the physics of the problem. In this particular case, they have to be located evenly along the length of the stub. Of interest is the fact 
that MSEs with an extremely limited number of coarse scale nodes often provide a sufficiently accurate framework for the prediction of the dispersion properties of the fundamental, low frequency wave modes.

\section{Wave propagation in structural lattices}

A second set of examples also shows the ability of the proposed MSE framework to be employed for the simulation of the propagation of waves in finite domains. This is illustrated for the case of structural lattices, which are known for their dynamic anisotropic properties that are related to the lattice topology. Such anisotropy leads to wave directionality, which can be exploited for wave guiding purposes $[6,46,47$, 48]. In this example, the GMsFEM is applied to compute the band diagrams and polar plots of wave velocities, which are evaluated through the unit cell approach described in [46]. Analysis are conducted using a GMsFEM discretization of the unit cell in which the influence of different MSEs is investigated through comparison with reference FEM solutions. Time domain simulations of the dynamic response of finite domains as assemblies of multiple unit cells emphasize the agreement between the GMsFEM and FEM solutions.

\subsection{Configuration}

A schematic of the considered configuration is shown in Fig. 13.a along with the corresponding unit cell in Fig. 13.b. The unit cell features side length $L=0.5 \mathrm{~mm}$ and isotropic ligaments $\left(E=71 \mathrm{GPa}, \rho=2700 \mathrm{~kg} / \mathrm{m}^{3}, \nu=0.3\right)$ oriented at $\theta=45 \mathrm{deg}$ with respect to the cartesian frame $\left(\boldsymbol{i}_{1}, \boldsymbol{i}_{2}\right)$.

According to Fig. 14.a, the unit cell is discretized by a single MSE featuring 88 global nodes positioned along the perimeter of the cell. In this example, several 
fine scale meshes (Figs. 14.b-d) are used to compute the MSE's mapping functions (eq. (7)) and evaluate their influence on the computed results.

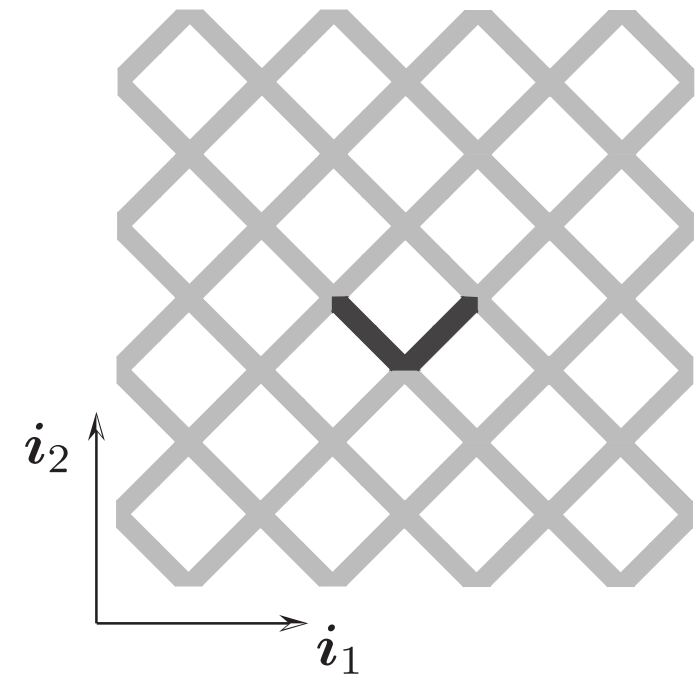

(a) Periodic lattice

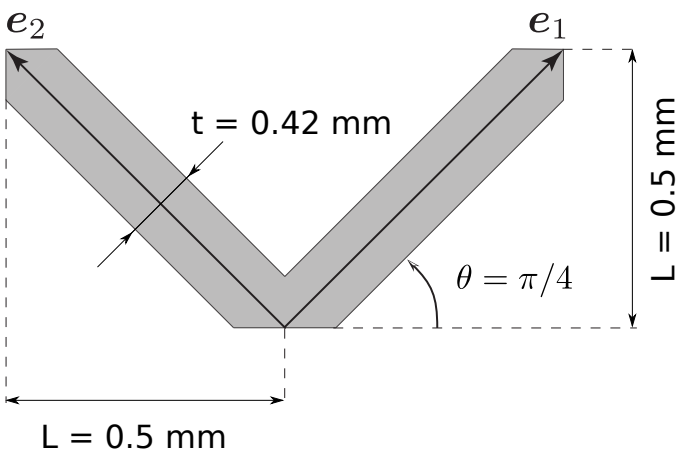

(b) Unit cell

Figure 13: Periodic two-dimensional lattice (a) and corresponding unit cell (b).

Of note is that the computational cost of GMsFEM simulations is mostly dictated by the number of global nodes defining the MSE, while the size of the auxiliary mesh has little influence on the overall computational time. As described in [35], the shape functions are in fact computed in the preprocessing stage of the simulation as the solution of a linear system of equations involving the fine scale DOFs. The computational cost of this operation performed, for example, on the fine scale triangulations shown in Figs. 14.b-d is almost negligible compared to the CPU time required by the subsequent time-domain or frequency analysis.

The influence of different fine scale meshes on the transient response of the finite domain system is investigated by comparing the GMsFEM predictions with FE results obtained using a refined discretization of the cell including 2121 nodes. Such 


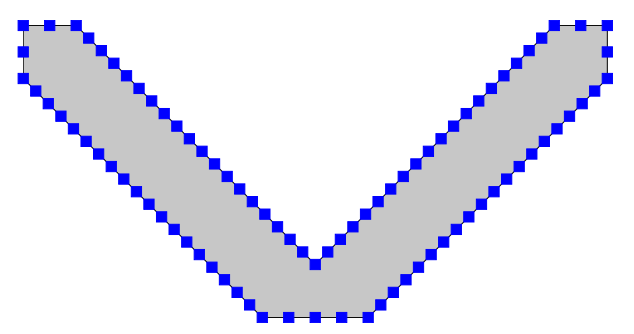

(a) MSE (88 global nodes)

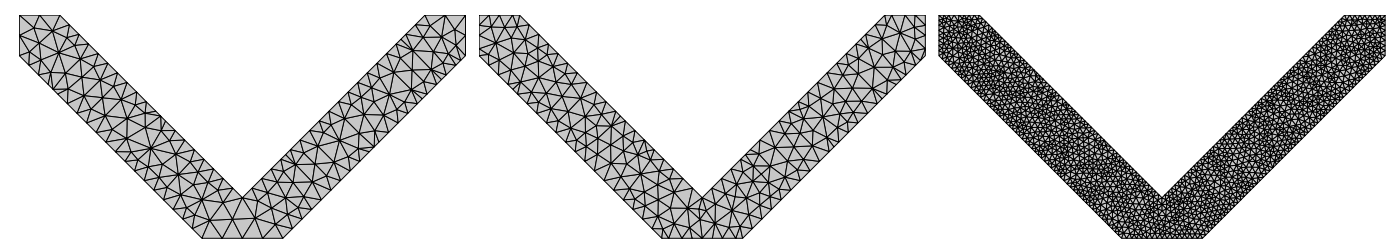

(b) mesh A (198 local nodes) (c) mesh B (753 local nodes) (d) mesh C (1595 local nodes)

Figure 14: Multiscale element used to discretize the unit cell (a), and different fine scale auxiliary meshes $(b, c, d)$ used for the calculation of the shape functions.

mesh is considered as sufficiently refined to provide converged estimations of the first five dispersion branches, which for the present configuration are characterized by a frequency upper limit of $4 \mathrm{MHz}$, as illustrated in the upcoming section.

\subsection{Band diagrams and wave velocities}

The cell's dispersion relations computed with the three GMsFEM models denoted as GMsFEM-A -B and -C in Fig. 14 are shown in Fig. 15. As previously done, the frequency range of analysis is arbitrarily set to include the first five wave modes. These results indicate that the adopted MSEs accurately predict the low-frequency region of the dispersion relations, while providing only an approximate representation of the higher portion of the considered frequency spectrum. Figure 15 also shows that the accuracy of the adopted MSE is modestly affected by the refinement of the corresponding fine scale mesh, which mostly influences the convergence of the dispersion branches at higher frequencies. 


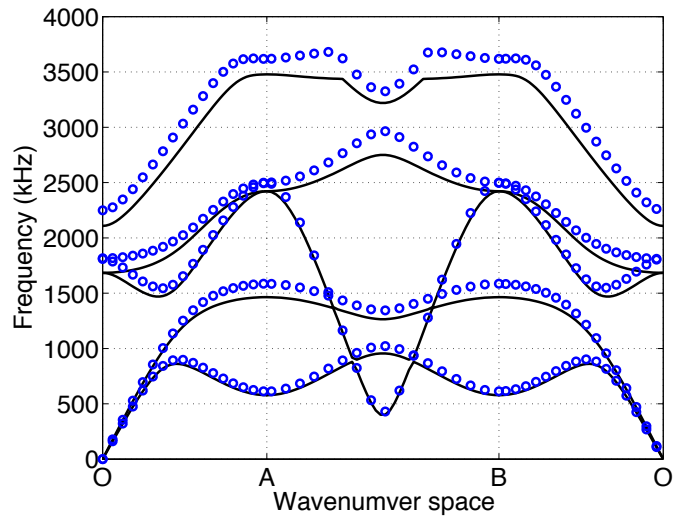

(a) GMsFEM-A

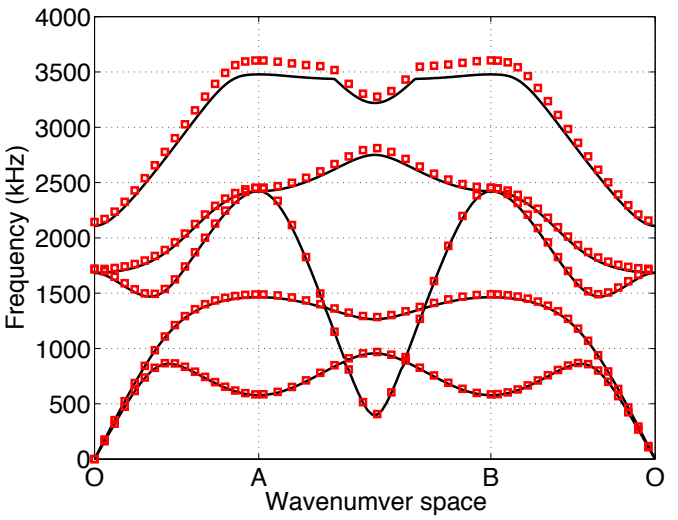

(b) GMsFEM-B

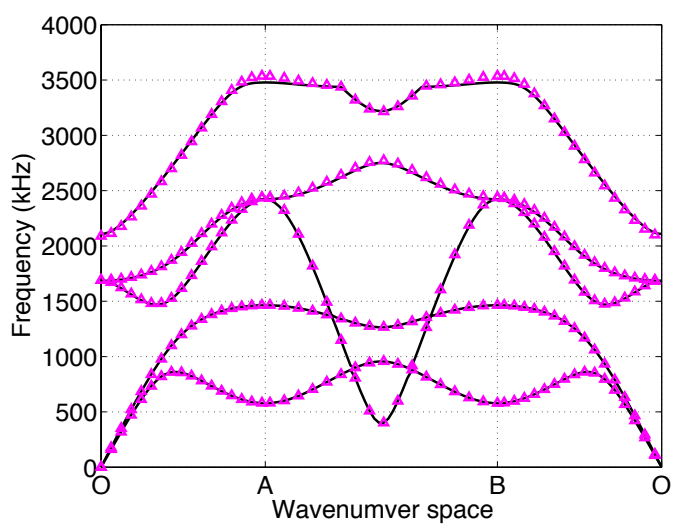

(c) GMsFEM-C

Figure 15: Dispersion relations computed with MSEs featuring different fine scale meshes (markers), and comparison with the reference FEM solution (thick solid line).

These results suggest that the GMsFEM can be particularly suited to investigate the dynamic behavior of this lattice configuration in the low-frequency / longwavelength regime where the system features strong wave directionality [46]. The directivity of the phase velocities in the long wavelength region in particular reflects the quasi-static anisotropy of the lattice, and describes the non-dispersive properties of the medium at low frequencies. Given the considered configuration, this quasi- 
static, non-dispersive regime extends to $0-500 \mathrm{kHz}$.

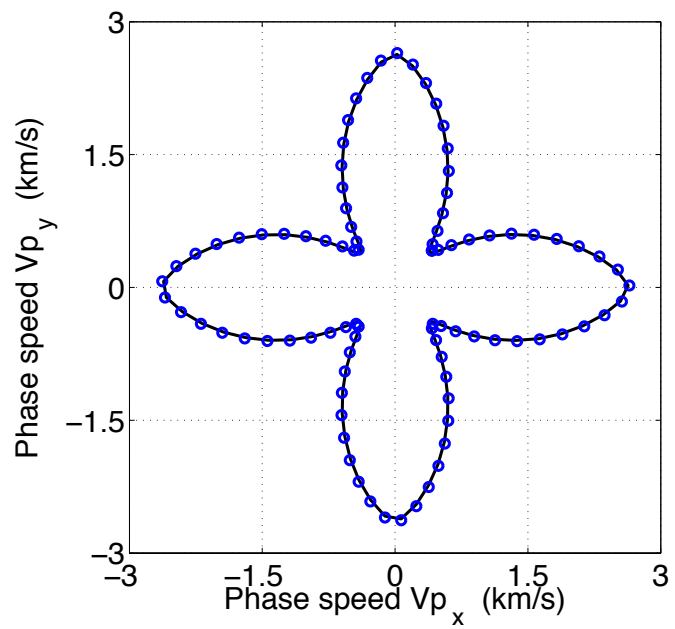

(a) Mode 1 (shear-like)

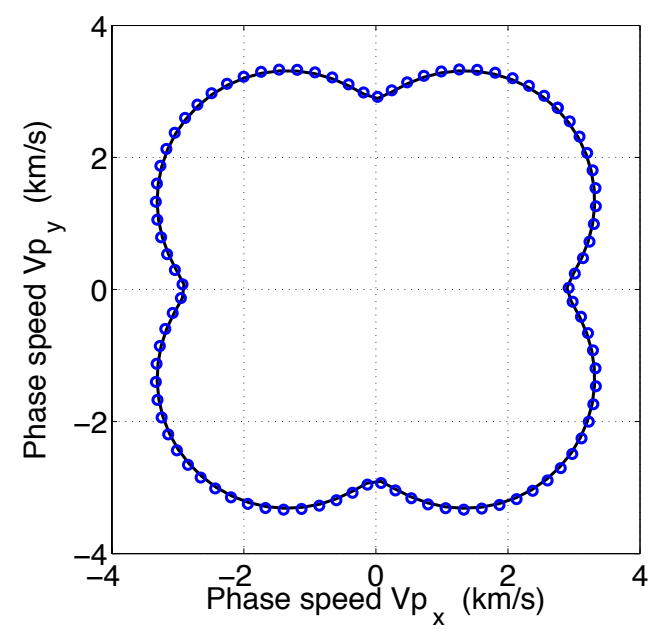

(b) Mode 2 (pressure-like)

Figure 16: Phase speed of the first (a) and second (b) wave modes computed with the reference FEM mesh (thick solid line) and with the GMsFEM-A mesh (o).

The phase velocities associated with the first two wave modes (the shear-like and the pressure-like modes) in the long wavelength regime are shown in Fig. 16. Results illustrate the excellent agreement between the refined FEM solution (thick solid line) and the GMsFEM solution obtained with 88 global nodes and the coarsest fine scale mesh (GMsFEM-A mesh).

The convergence history of the wave velocities at long wavelengths is also investigated in order to compare the GMsFEM and FEM methods. The analysis is conducted in terms of the following percent error

$$
\mathcal{E}=\frac{\left|v_{p h}^{e x}-v_{p h}^{n u m}\right|}{\left|v_{p h}^{e x}\right|} \cdot 100
$$

where $v_{p h}^{e x}$ and $v_{p h}^{\text {num }}$ respectively denote the exact and numerical phase velocities computed as a function of the number of coarse scale DOFs retained in the FEM and 
GMsFEM models. Results in Fig. 17 indicate that both the GMsFEM and FEM

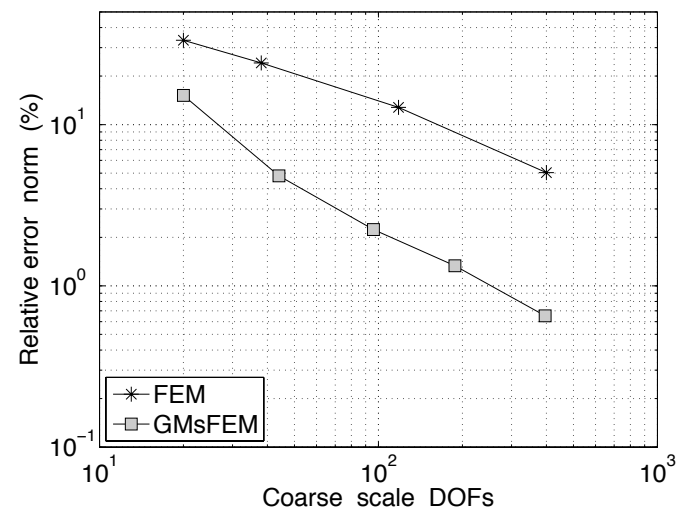

(a) Mode 1 (shear-like)

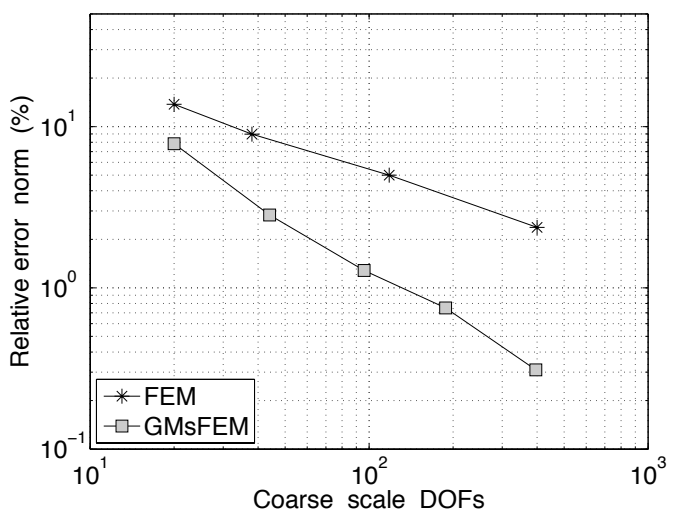

(b) Mode 2 (pressure-like)

Figure 17: Convergence of the phase velocity error $\mathcal{E}$ associated with GMsFEM and FEM for increasing coarse scale DOFs.

solutions tend to the reference one for increasing mesh refinements, and demonstrate that the proposed GMsFEM is characterized by smaller errors compared to a traditional FE discretization featuring a similar number of macroscopic DOFs. As discussed in detail in [35], this is attributed to the fact that the GMsFEM basis functions are tailored to the local solution of the problem for vanishing frequencies.

\subsection{Transient response of a finite assembly}

The comparison between GMsFEM and FEM simulations is further conducted in terms of time domain simulations involving a finite periodic assembly composed of 15 unit cells along the $x$ and $y$ directions. The structure is excited by a narrowband 10-cycle tone burst signal centered at a frequency of $100 \mathrm{kHz}$ as shown in Fig. 18.b. The frequency spectrum of the applied load is limited to the frequency region where the dynamic response is governed by the superposition of the two fundamental wave modes whose phase velocities are shown in Fig. 16. Four concentrated forces are 


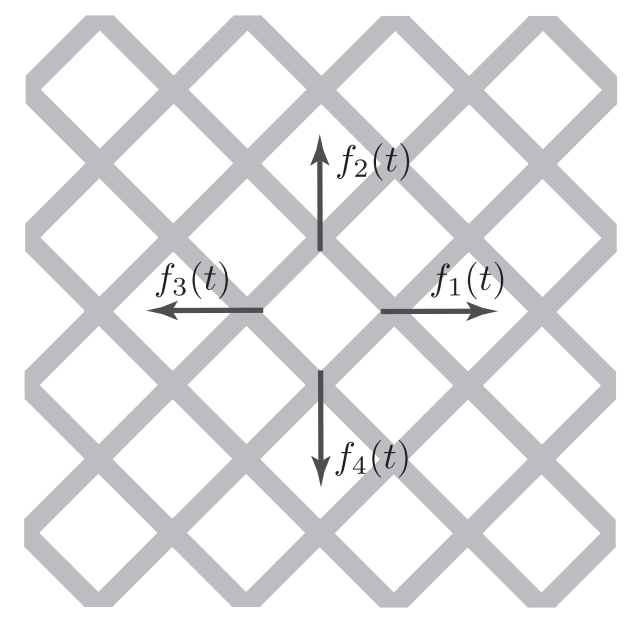

(a)

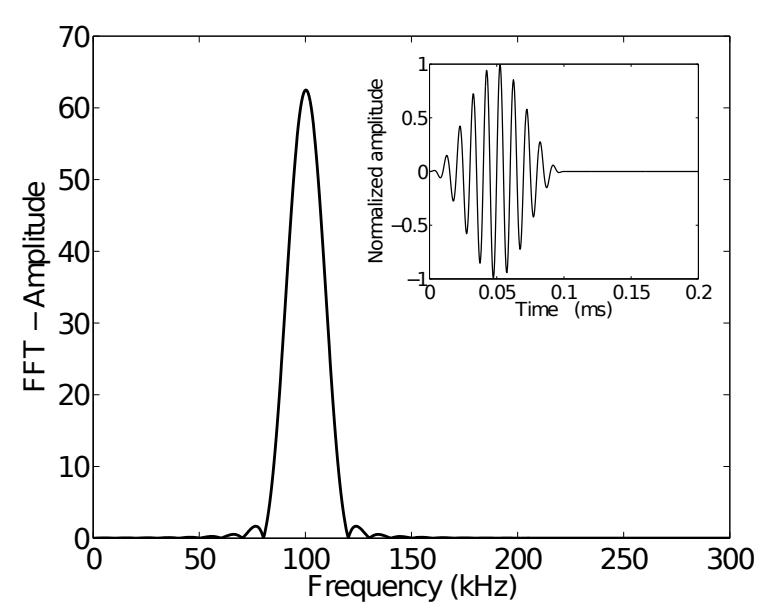

(b)

Figure 18: Spatial arrangement (a), and time variation (b) of the nodal forces applied to the lattice.

simultaneously applied at the nodal points of the central cell to uniformly excite the structure in multiple directions (see Fig. 18.a). The response is evaluated by integrating the equations of motion of the assembly with a time marching scheme of the Newmark family [49] using free-displacement boundary conditions at the edges of the computational domain.

Results presented in Fig. 19 illustrate snapshots of the displacement field captured before observing reflections from the boundaries. Interestingly, the GMsFEM allows for integration of the dynamic equations of the problem in about $5.5 \mathrm{sec}$, while $36 \mathrm{sec}$ are required to integrate the FE system. Such CPU times are evaluated considering 200 time-steps, and using the same implementation of the time marching scheme. Clearly, GMsFEM is more advantageous since a smaller number of DOFs is explicitly retained throughout the entire time marching loop. Results are presented side-byside to emphasize the agreement between the GMsFEM-A and the refined FEM solutions. As predicted by the unit cell analysis, the system is characterized by a 
significant directional behavior which is fully captured by the GMsFEM scheme.

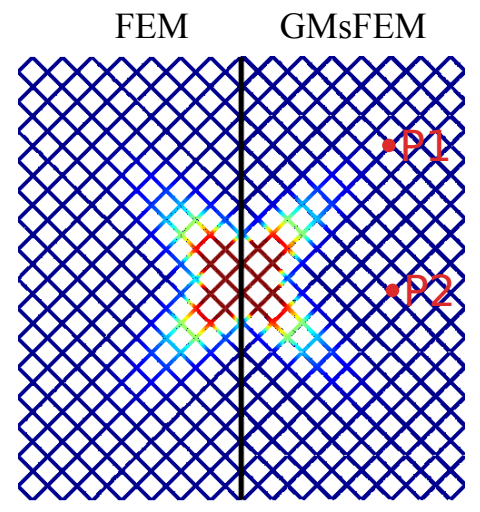

(a)

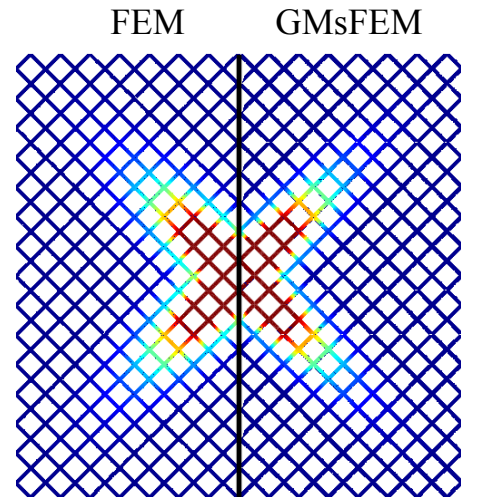

(b)

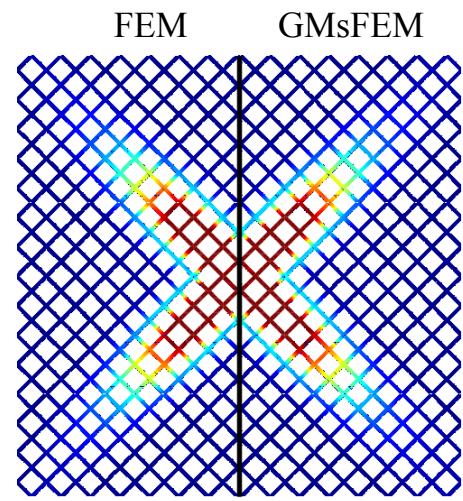

(c)

Figure 19: Comparison between the FEM and GMsFEM displacement fields for evolving time-steps (amplitude is normalized to unity).

A similar agreement between the two sets of results is also evident in Fig. 20 which shows the time variation of the components of the displacement vector at two representative points denoted as $\mathrm{P} 1$ and P2 in Fig. 19.a.

These results confirm that convergence analysis of the band diagrams or phase velocities on the unit cell prove to be a very efficient way of assessing the convergence of a particular GMsFEM discretization. The resulting GMsFEM mesh can later be used for problems involving the transient response of finite periodic structures for which estimation of convergence would be a computationally intensive task.

\section{Conclusions}

This paper illustrates the application of the geometric multiscale finite element method for the analysis of wave propagation in heterogeneous periodic solids. The approach allows modeling the unit cell by means of one MSE characterized by a 


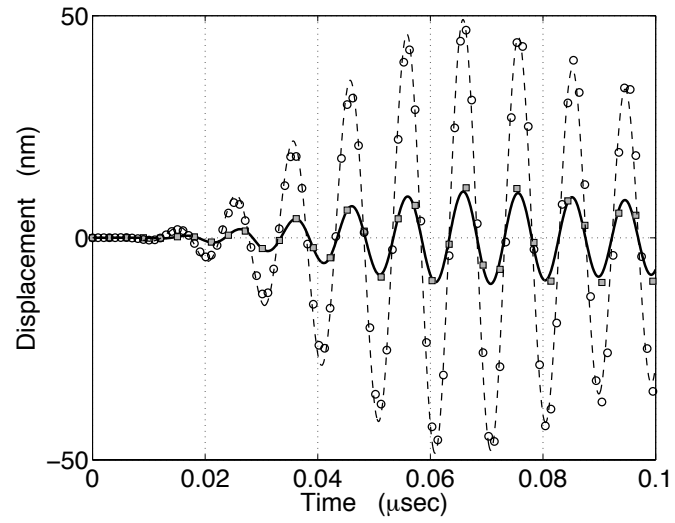

(a) Displacement $u_{1}$

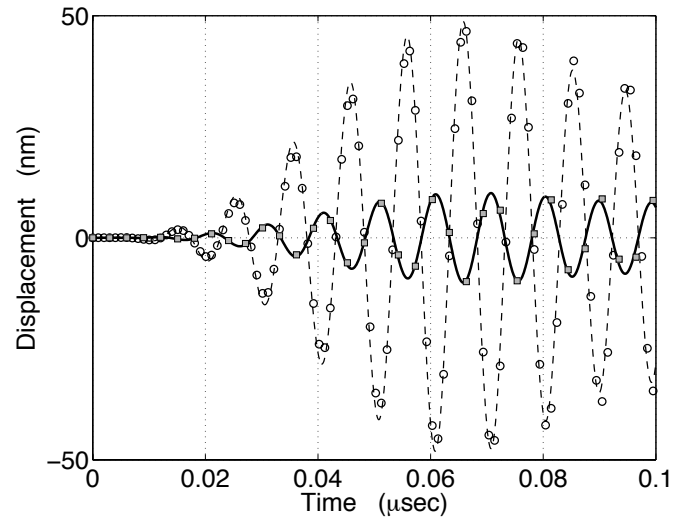

(b) Displacement $u_{2}$

Figure 20: Comparison between the FEM (lines) and the GMsFEM (markers) time histories computed at point P1 (thin dashed line) and P2 (thick solid line) - defined in Fig. 19.a

limited number of global nodes. Two- and three-dimensional multiscale elements are adopted to study the dispersion properties of, and transient wave propagation in periodic elastic domains. Band diagram calculations, wave velocities and time domain computations are conducted on solids discretized using two-dimensional and threedimensional multiscale finite element meshes. Convergence of a particular GMsFEM discretization is studied from the convergence analysis of the band diagrams or phase velocities of the unit cell.

Results indicate that extremely coarse MSEs can accurately predict the response of the system in the low frequency range where the motion of the unit cell is characterized by global deformation patterns. If a higher frequency range is of interest, the present method could be extended through the addition of shape functions corresponding to the natural vibration modes of the MSE [50]. In this case, there is some some resemblance to methods that use component mode synthesis, for example the Craig-Bampton method. 
The proposed method allows to seamlessly extend the frequency range of accuracy of one MSE by increasing the number of global nodes which must be selected to capture the physics of the problem. Numerical results also show that the specific fine scale discretization adopted for the calculation of the shape functions has a modest influence on the accuracy of the macroscopic computation, and that its effects are mostly affecting the convergence of the dispersion branches at higher frequencies. Transient simulations conducted for finite-size periodic assemblies corroborate the agreement between GMsFEM and FEM results predicted by the unit cell analysis.

\section{Aknowledgements}

The authors would like to thank the National Science Foundation for supporting this research through the CMMI grant 1130368. 


\section{References}

[1] I. E. Psarobas, Phononic crystals: sonic band-gap materials, Zeitschrift fur Kristallographie, 2005.

[2] R. Langley, The response of two-dimensional periodic structures to point harmonic forcing, Journal of sound and vibration 197 (4) (1996) 447-469.

[3] A. Movchan, N. Movchan, S. Haq, Localised vibration modes and stop bands for continuous and discrete periodic structures, Materials Science and Engineering: A 431 (1-2) (2006) 175-183.

[4] M. Ruzzene, F. Scarpa, Directional and band-gap behavior of periodic auxetic lattices, physica status solidi (b) 242 (3) (2005) 665-680.

[5] M. Ruzzene, F. Scarpa, F. Soranna, Wave beaming effects in two-dimensional cellular structures, Smart materials and structures 12 (2003) 363.

[6] A. Spadoni, M. Ruzzene, S. Gonella, F. Scarpa, Phononic properties of hexagonal chiral lattices, Wave Motion 46 (7) (2009) 435-450.

[7] E. Plum, J. Zhou, J. Dong, V. Fedotov, T. Koschny, C. Soukoulis, N. Zheludev, Metamaterial with negative index due to chirality, Physical Review B 79 (3) (2009) 035407.

[8] A. Sukhovich, L. Jing, J. Page, Negative refraction and focusing of ultrasound in two-dimensional phononic crystals, Physical Review B 77 (1) (2008) 014301.

[9] M. Senesi, B. Xu, M. Ruzzene, Experimental characterization of periodic frequency-steerable arrays for structural health monitoring, Smart Materials and Structures 19 (2010) 055026. 
[10] F. Casadei, M. Ruzzene, L. Dozio, K. Cunefare, Broadband vibration control through periodic arrays of resonant shunts: experimental investigation on plates, Smart Materials and Structures 19 (2010) 015002.

[11] J. Wolfe, Imaging phonons: acoustic wave propagation in solids, Cambridge Univ Pr, 1998.

[12] S. Cox, D. Dobson, Band structure optimization of two-dimensional photonic crystals in h-polarization, Journal of Computational Physics 158 (2) (2000) 214224 .

[13] H. Men, N. Nguyen, R. Freund, P. Parrilo, J. Peraire, Bandgap optimization of two-dimensional photonic crystals using semidefinite programming and subspace methods, Journal of Computational Physics 229 (10) (2010) 3706-3725.

[14] N. Bakhvalov, G. Panasenko, M. Eglit, The effective properties of structures and composites with inclusions in the form of walls and rods, Computational mathematics and mathematical physics 36 (12) (1996) 1701-1706.

[15] A. Bensoussan, J. Lions, G. Papanicolaou, Asymptotic analysis for periodic structures, Vol. 5, North Holland, 1978.

[16] J. Lions, Some methods of resolution of free surface problems, in: Proceedings of the Fifth International Conference on Numerical Methods in Fluid Dynamics June 28-July 2, 1976 Twente University, Enschede, Springer, 1976, pp. 1-31.

[17] E. Palencia, Non-homogeneous media and vibration theory, Springer-Verlag, 1980.

[18] L. Gibson, M. Ashby, Cellular solids: structure and properties, Cambridge Univ Pr, 1999. 
[19] I. Babuska, The computational aspects of the homogenization problem, Computing Methods in Applied Sciences and Engineering, 1977, I (1979) 309-316.

[20] P. Martinsson, Fast multiscale methods for lattice equations, Ph.D. thesis, University of texas at Austin (2002).

[21] P. Martinsson, A. Movchan, Vibrations of lattice structures and phononic band gaps, The Quarterly Journal of Mechanics and Applied Mathematics 56 (1) (2003) 45.

[22] P. Martinsson, G. Rodin, Asymptotic expansions of lattice green's functions, Proceedings of the Royal Society of London. Series A: Mathematical, Physical and Engineering Sciences 458 (2027) (2002) 2609.

[23] J. Oden, K. Vemaganti, N. Moës, Hierarchical modeling of heterogeneous solids, Computer Methods in Applied Mechanics and Engineering 172 (1-4) (1999) 325 .

[24] J. Fish, V. Belsky, Multi-grid method for periodic heterogeneous media part 2: Multiscale modeling and quality control in multidimensional case, Computer Methods in Applied Mechanics and Engineering 126 (1-2) (1995) 17-38.

[25] J. Fish, W. Chen, Higher-order homogenization of initial/boundary-value problem, Journal of engineering mechanics 127 (12) (2001) 1223-1230.

[26] J. Fish, W. Chen, Rve-based multilevel method for periodic heterogeneous media with strong scale mixing, Journal of engineering mathematics 46 (1) (2003) 87106. 
[27] J. Fish, W. Chen, G. Nagai, Non-local dispersive model for wave propagation in heterogeneous media: multi-dimensional case, International Journal for $\mathrm{Nu}-$ merical Methods in Engineering 54 (3) (2002) 347-363.

[28] J. Fish, W. Chen, G. Nagai, Non-local dispersive model for wave propagation in heterogeneous media: one-dimensional case, International Journal for Numerical Methods in Engineering 54 (3) (2002) 331-346.

[29] J. Fish, A. Wagiman, Multiscale finite element method for a locally nonperiodic heterogeneous medium, Computational mechanics 12 (3) (1993) 164-180.

[30] H. Murakami, A mixture theory for wave propagation in angle-ply laminates, part 1: theory, Journal of applied mechanics 52 (1985) 331.

[31] H. Murakami, A. Akiyama, A mixture theory for wave propagation in angle-ply laminates, part 2: application, Journal of applied mechanics 52 (1985) 338.

[32] H. Murakami, G. Hegemier, A mixture model for unidirectionally fiberreinforced composites, Journal of applied mechanics 53 (1986) 765.

[33] M. Hussein, Reduced Bloch mode expansion for periodic media band structure calculations, Proceedings of the Royal Society A: Mathematical, Physical and Engineering Science 465 (2109) (2009) 2825-2848.

[34] H. Brandsmeier, K. Schmidt, C. Schwab, A multiscale hp-fem for 2d photonic crystal bands, Journal of Computational Physics 230 (2) (2011) 349-374.

[35] F. Casadei, J.J. Rimoli, M. Ruzzene, A geometric multiscale finite element method for the dynamic analysis of heterogeneous solids, Computer Methods in Applied Mechanics and Engineering 263 (2013) 56-70. 
[36] F. Casadei, J.J. Rimoli, M. Ruzzene, Multiscale finite element analysis of elastic wave scattering from localized defects, Finite Elements in Analysis and Design 88 (2014) $1-15$.

[37] L. Brillouin, Wave propagation in periodic structures electric filters and crystal lattices, Dover Publications Inc., 1946.

[38] C. Kittel, P. McEuen, Introduction to solid state physics, Vol. 7, Wiley New York, 1976.

[39] F. Vernerey, M. Kabiri, An adaptive concurrent multiscale method for microstructured elastic solids, Computer Methods in Applied Mechanics and Engineering.

[40] A. Khelif, B. Aoubiza, S. Mohammadi, A. Adibi, V. Laude, Complete band gaps in two-dimensional phononic crystal slabs, Physical Review E 74 (4) (2006) 046610.

[41] S. Mohammadi, A. Eftekhar, A. Khelif, W. Hunt, A. Adibi, Evidence of large high frequency complete phononic band gaps in silicon phononic crystal plates, Applied Physics Letters 92 (22) (2008) 221905-221905.

[42] Y. Pennec, B. Djafari-Rouhani, H. Larabi, J. Vasseur, A. Hladky-Hennion, Lowfrequency gaps in a phononic crystal constituted of cylindrical dots deposited on a thin homogeneous plate, Physical Review B 78 (10) (2008) 104105.

[43] T. Wu, Z. Huang, T. Tsai, T. Wu, Evidence of complete band gap and resonances in a plate with periodic stubbed surface, Applied Physics Letters 93 (2008) 111902. 
[44] T.-C. Wu, T.-T. Wu, J.-C. Hsu, Waveguiding and frequency selection of lamb waves in a plate with a periodic stubbed surface, Phys. Rev. B 79 (2009) 104306.

[45] W. Bragg, The diffraction of short electromagnetic waves by a crystal, in: Proceedings of the Cambridge Philosophical Society, Vol. 17, 1913, pp. 43-57.

[46] F. Casadei, J. Rimoli, Anisotropy-induced broadband stress wave steering in periodic lattices, International Journal of Solids and Structures.

[47] S. Gonella, M. Ruzzene, Analysis of in-plane wave propagation in hexagonal and re-entrant lattices, Journal of Sound and Vibration 312 (1) (2008) 125-139.

[48] A. Phani, J. Woodhouse, N. Fleck, Wave propagation in two-dimensional periodic lattices, J. Acoustical Soc. Am. 4 (119) (2006) 1995-2005.

[49] N. Newmark, A method of computation for structural dynamics, J. Eng. Mech. 85 (1959) 67-94.

[50] G. Capuano, M. Ruzzene, J. J. Rimoli, Modal-based finite elements for efficient wave propagation analysis, Wave Motion, Submitted. 